\title{
On Bost-Connes type systems and complex multiplication
}

\author{
Bora Yalkinoglu
}

\begin{abstract}
By using the theory of complex multiplication for general Siegel modular varieties we construct arithmetic subalgebras for BC-type systems attached to number fields containing a $\mathrm{CM}$ field. The abelian extensions obtained in this way are characterized by results of [Wei]. Our approach is based on a general construction of BC-type systems of Ha and Paugam [HP05] and extends the construction of the arithmetic subalgebra of Connes, Marcolli and Ramachandran [CMR05] for imaginary quadratic fields.
\end{abstract}

Mathematics Subject Classification (2010). 58B34, 11R37, 14G35.

Keywords. Bost-Connes type systems, complex multiplication, Shimura varieties.

\section{Contents}

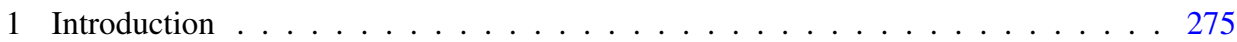

2 On the arithmetic subalgebra for $K=\mathbb{Q}(i) \ldots \ldots \ldots \ldots \ldots$. . . . . . . . . . . . . . . . . . . . .

3 Two Shimura data and a map . . . . . . . . . . . . . . . . . . . 290

4 About arithmetic modular functions . . . . . . . . . . . . . . . . . . . . 293

5 On Bost-Connes-Marcolli systems . . . . . . . . . . . . . . . . . . . . . . . . . . 298

6 Two BCM pairs and a map . . . . . . . . . . . . . . . . . . . . 301

7 Construction of our arithmetic subalgebra . . . . . . . . . . . . . 306

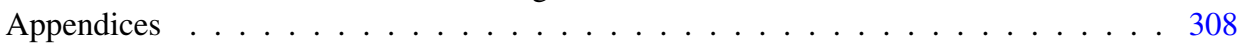

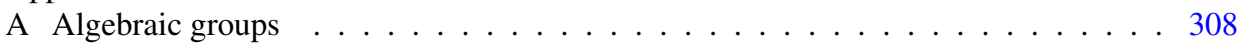

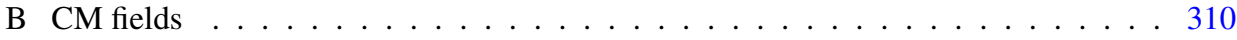

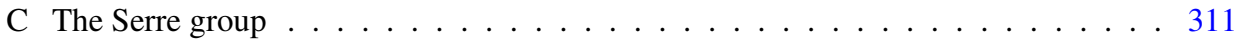

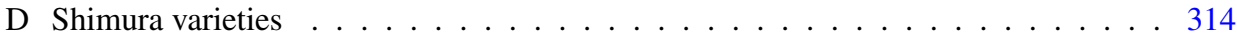

References . . . . . . . . . . . . . . . . . . . 318

\section{Introduction}

In their fundamental article [BC95] Bost and Connes constructed a quantum statistical mechanical system, the so-called Bost-Connes or BC system, that recovers (among other arithmetic properties of $\mathbb{Q}$ ) the explicit class field theory of $\mathbb{Q}$. It is a natural question to ask for other quantum statistical mechanical systems that recover - at least partially - the (explicit) class field theory of number fields different from $\mathbb{Q}$. 
This paper is a contribution to this question in the case of $\mathrm{CM}$ fields. In nature a $\mathrm{CM}$ field arises as (rational) endomorphism ring of an Abelian variety with complex multiplication.

Background. A quantum statistical mechanical system $\mathcal{A}=\left(A,\left(\sigma_{t}\right)_{t \in \mathbb{R}}\right)$ is a $C^{*}$ algebra $A$ together with a one-parameter group of automorphisms $\left(\sigma_{t}\right)_{t \in \mathbb{R}}$. Sometimes we call $\mathcal{A}$ simply a $C^{*}$-dynamical system. One should think of $A$ as the algebra of observables of a quantum physical system with the one-parameter group $\left(\sigma_{t}\right)_{t \in \mathbb{R}}$ implementing the time evolution of the observables. A symmetry of $\mathcal{A}$ is a $C^{*}$ automorphism of $A$ which commutes with the time evolution. Interesting properties of $\mathcal{A}$ can be read off its equilibrium states at a temperature $T \in[0, \infty)$. An equilibrium state at inverse temperature $\beta=\frac{1}{T}$ is given by a so-called $\mathrm{KMS}_{\beta}$-state. See [BR79] and [BR81] for more information.

Now a first step towards generalizing the Bost-Connes system to other number fields is given by a system of the following kind (see [CMR06] and [LLN09]):

A BC-type system for a number field $K$ is a quantum statistical mechanical system $\mathcal{A}=\left(A,\left(\sigma_{t}\right)_{t \in \mathbb{R}}\right)$ with the following properties:

(i) The partition function of $\mathcal{A}$ is given by the Dedekind zeta function of $K$.

(ii) The quotient of the idele class group $C_{K}$ by the connected component $D_{K}$ of the identity of $C_{K}$ acts as symmetries on $\mathcal{A}$.

(iii) For each inverse temperature $0<\beta \leq 1$ there is a unique $\mathrm{KMS}_{\beta}$-state.

(iv) For each $\beta>1$ the action of the symmetry group $C_{K} / D_{K}$ on the set of extremal $\mathrm{KMS}_{\beta}$-states is free and transitive.

Remark. i) Using class field theory we see that property (ii) simply states that the Galois group $\operatorname{Gal}\left(K^{\mathrm{ab}} / K\right) \cong C_{K} / D_{K}$ of the maximal abelian extension $K^{\mathrm{ab}}$ of $K$ is acting by symmetries on $\mathcal{A}$.

ii) Properties (iii) and (iv) say that there is a spontaneous symmetry breaking phenomenon at $\beta=1$ (see [BC95] or p. $400 \mathrm{ff}$. in the book [CM08] by Connes and Marcolli).

Due to the work of Ha and Paugam [HP05] BC-type systems are known to exist for an arbitrary number field $K$, we denote their solution (see the next section for the definition) by

$$
\mathcal{A}_{K}=\left(A_{K},\left(\sigma_{t}\right)_{t \in \mathbb{R}}\right) .
$$

The paper of Laca, Larsen and Neshveyev [LLN09] gives a different description of $\mathcal{A}_{K}$. In fact [HP05] yields that $\mathcal{A}_{K}$ fulfills the first two properties and [LLN09] verifies the last two properties.

We should mention that the approach of [HP05] works much more general, it allows to attach a quantum statistical mechanical system to an arbitrary Shimura variety, the above construction being a special case. The Shimura-theoretic approach 
of [HP05] is based on the foundational work of Connes, Marcolli and Ramachandran (see [CMR05] and [CMR06]).

The notion of an arithmetic subalgebra for a BC-type system, first encountered in [BC95], hints to a possible relation between seemingly unrelated areas, namely it asks for a connection between quantum statistical mechanical systems (physics/operator algebras) and class field theory (number theory). More precisely (see [CMR06]), a $B C$-system for a number field $K$ is a BC-type system $\mathcal{A}=\left(A,\left(\sigma_{t}\right)_{t \in \mathbb{R}}\right)$ such that:

(v) There is a $K$-rational subalgebra $A^{\text {arith }}$ of $A$, called arithmetic subalgebra of $\mathcal{A}$, such that for every extremal $\mathrm{KMS}_{\infty}$-state $\varrho$ and every $f \in A^{\text {arith }}$ we have

$$
\varrho(f) \in K^{\mathrm{ab}}
$$

and further $K^{\mathrm{ab}}$ is generated over $K$ in this way, i.e.,

$$
K^{\mathrm{ab}}=K\left(\varrho(f) \mid \varrho \text { extremal } \mathrm{KMS}_{\infty} \text {-state, } f \in A^{\text {arith }}\right) .
$$

(vi) Let $v \in C_{K}$ be a symmetry of $\mathcal{A}, \varrho$ an extremal $\mathrm{KMS}_{\infty}$-state of $\mathcal{A}$ and $f \in$ $A^{\text {arith }}$. Denote by $[v]$ the image of $v$ under Artin's reciprocity morphism $C_{K} \rightarrow$ $\operatorname{Gal}\left(K^{\mathrm{ab}} / K\right)$ and by ${ }^{v} \varrho$ the action (given by pull-back) of $v$ on $\varrho$. Then we have the compatibility relation

$$
{ }^{\nu} \varrho(f)=[\nu]^{-1}(\varrho(f)) \in K^{\mathrm{ab}} .
$$

Now the difficulty of constructing BC-systems comes from its relation with Hilbert's twelfth problem, which asks for an explicit class field theory of a number field $K$. This problem is completely solved only in two cases, namely the case of $K=\mathbb{Q}$ and the case of $K$ equal to an imaginary quadratic field, like $K=\mathbb{Q}(i)$. It does not come as a surprise that BC-systems are so far known to exist only in these two cases. For $K=\mathbb{Q}$ see [BC95] and for the imaginary quadratic case we refer to [CMR05] and the very detailed exposition given in [CM08], p. $551 \mathrm{ff}$.

The construction in [CMR05] is based on the theory of complex multiplication (see Section 4) which is a part of the arithmetic theory of Shimura varieties. This theory allows to construct explicitly abelian extensions of higher-dimensional generalizations of imaginary quadratic number fields, so-called CM fields. A CM field $E$ is a totally imaginary quadratic extension of a totally real number field. So, for example, cyclotomic number fields $\mathbb{Q}\left(\zeta_{n}\right), \zeta_{n}$ being a primitive $n$-th root of unity, are seen to be CM-fields.

Abelian extensions of a CM field $E$ are obtained by evaluating arithmetic modular functions on so-called CM-points on a Siegel upper half plane (see Section 4.1 for more information).

Except for the case of an imaginary quadratic field, it is unfortunately not possible to generate the maximal abelian extension $E^{\mathrm{ab}}$ of a $\mathrm{CM}$ field $E$ in this way, but still a non-trivial abelian extension of infinite degree over $E$. We denote the latter by

$$
E^{c} \subset E^{\mathrm{ab}} .
$$


See Theorem 4.2 for the characterization of $E^{c}$ given in [Wei]. As we remarked above, for $E$ equal to an imaginary quadratic field there is an equality $E^{c}=E^{\text {ab }}$.

Due to the lack of a general knowledge of the explicit class field theory of an arbitrary number field $K$ we content ourself with the following weakening of a BCsystem:

Let $F \subset K^{\text {ab }}$ be an arbitrary abelian extension of $K$. A partial BC-system for the extension $F / K$ is defined like a BC system $\mathcal{A}=\left(A,\left(\sigma_{t}\right)_{t \in \mathbb{R}}\right)$ for $K$ except that we do not demand the arithmetic subalgebra $A^{\text {arith }}$ of $A$ to generate the maximal abelian extension $K^{\mathrm{ab}}$ of $K$ but instead the abelian extension of $F$, i.e., in property (v) we replace $K^{\mathrm{ab}}$ with $F$ and call the corresponding $K$-rational subalgebra $A^{\text {arith }}$ a (partial) arithmetic subalgebra of $\mathcal{A}$.

Statement of our result. Now the aim of our paper is to prove the following result.

Theorem 1.1. Let $K$ be a number field containing a CM field. Denote by $E$ the maximal $C M$ field contained in $K$ and define the abelian extension $K^{c}$ of $K$ to be the compositum

$$
K^{c}=K \cdot E^{c}
$$

(with the notation from (2)).

Then the BC-type system $\mathcal{A}_{K}$ of [HP05] (see (6.1)) is a partial BC-system for the extension $K^{c} / K$, i.e., there exists a (partial) arithmetic subalgebra $A_{K}^{\text {arith }}$ for $\mathcal{A}_{K}$.

We want to explain our construction of $A_{K}^{\text {arith }}$ (cf. Section 7). It is inspired by the construction given in [CMR05] and [CMR06] (see also [CM08], p. 551 ff.).

Idea of our construction. Let $(G, X, h)$ be a Shimura datum and denote by $\operatorname{Sh}(G, X, h)$ the associated Shimura variety. In [HP05] the authors associate to the datum $(G, X, h)$, the variety $\operatorname{Sh}(G, X, h)$ and some additional data a quotient map

$$
U \rightarrow Z
$$

between a topological groupoid $U$ and a quotient $Z=\Gamma \backslash U$ for a group $\Gamma$. Out of this data they construct a $C^{*}$-dynamical system $\mathcal{A}=\left(A,\left(\sigma_{t}\right)_{t \in \mathbb{R}}\right)$. A dense $*$-subalgebra $H$ of $A$ is thereby given by the compactly supported, continuous functions

$$
H=C_{c}(Z)
$$

on $Z$, where the groupoid structure of $U$ induces the $*$-algebra structure on $H$.

Moreover there are two variations of the above quotient map which can be put 
into the following (commutative) diagram:

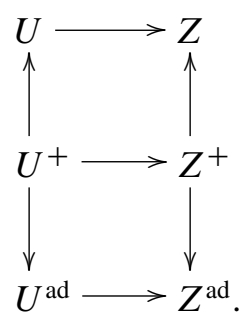

For more information see Section 5. We will apply this general procedure in two special cases. For this we fix a number field $K$ together with a maximal CM subfield $E$.

I) The BC-type system $\mathscr{A}_{\boldsymbol{K}}$. The (0-dimensional) Shimura datum

$$
\varsigma_{K}=\left(T^{K}, X_{K}, h_{K}\right)
$$

gives rise to a quotient map denoted by

$$
U_{K} \rightarrow Z_{K}
$$

In this case the groupoid $U_{K}$ is of the form (cf. (4))

$$
U_{K}=T^{K}\left(\mathbb{A}_{f}\right) \boxplus\left(\widehat{\mathcal{O}}_{K} \times \operatorname{Sh}\left(T^{K}, X_{K}, h_{K}\right)\right) .
$$

The associated $C^{*}$-dynamical system is denoted by

$$
\mathcal{A}_{K}=\left(A_{K},\left(\sigma_{t}\right)_{r \in \mathbb{R}}\right) \text {. }
$$

It gives rise to a BC-type system for $K$. For the precise definition of $\mathcal{A}_{K}$ and its properties (e.g. symmetries, extremal $\mathrm{KMS}_{\infty}$-states) we refer the reader to Sections 3.1 and 6.1. Moreover we denote by $H_{K}$ the dense subalgebra of $A_{K}$ given by

$$
H_{K}=C_{c}\left(Z_{K}\right)
$$

II) The Shimura system $\mathscr{A}_{\mathrm{Sh}}$. To the CM field $E$ we associate the Shimura datum

$$
\delta_{\mathrm{Sh}}=\left(\mathrm{GSp}\left(V_{E}, \psi_{E}\right), \mathbb{U}_{g}^{ \pm}, h_{\mathrm{cm}}\right),
$$

where in fact the construction of the morphism $h_{\mathrm{cm}}$ takes some time (see Section 3.2). This is due to two difficulties that arise in the case of a general CM field $E$ which are not visible in the case of imaginary quadratic fields. On the one hand one has to use the Serre group $S^{E}$, and on the other hand in general the reflex field $E^{*}$ of a CM field $E$ is not anymore equal to $E$ (see B.3). We denote the associated quotient map by

$$
U_{\mathrm{Sh}} \rightarrow Z_{\mathrm{Sh}}
$$


and analogously its variations (see Section 6.2.2). Here the relevant groupoids at hand are of the form

$$
U_{\mathrm{Sh}}=\operatorname{GSp}\left(\mathbb{A}_{f}\right) \boxplus\left(\Gamma_{\mathrm{Sh}, M} \times \operatorname{Sh}\left(\mathrm{GSp}\left(V_{E}, \psi_{E}\right), \mathbb{U}_{g}^{ \pm}, h_{\mathrm{cm}}\right)\right)
$$

and

$$
U_{\mathrm{Sh}}^{\mathrm{ad}}=\mathrm{GSp}^{\mathrm{ad}}(\mathbb{Q})^{+} \boxplus\left(\Gamma_{\mathrm{Sh}, M}^{\mathrm{ad}} \times \mathbb{H}_{g}\right) .
$$

We denote the resulting $C^{*}$-dynamical system by $\mathcal{A}_{\mathrm{Sh}}$ and call it Shimura system (cf. Section 5.3).

Remark. In the case of an imaginary quadratic field $K$ the Shimura system $\mathcal{A}_{\text {Sh }}$ gives rise to the $\mathrm{GL}_{2}$-system of Connes and Marcolli [CM08].

The second system is of great importance for us because of the following: Denote by $x_{\mathrm{cm}} \in \mathbb{U}_{g}$ the CM-point associated with $h_{\mathrm{cm}}$ and denote by $\mathcal{M}^{\mathrm{cm}}$ the ring of arithmetic modular functions on $\mathbb{U}_{g}$ defined at $x_{\mathrm{cm}}$. By the theory of complex multiplication we know that for every $f \in \mathcal{M}^{\mathrm{cm}}$ we have (cf. (3) and Section 4.3.1)

$$
f\left(x_{\mathrm{cm}}\right) \in K^{c} \subset K^{\mathrm{ab}}
$$

and moreover $K^{c}$ is generated in this way. Our idea is now that $\mathcal{M}^{\mathrm{cm}}$ gives rise to the arithmetic subalgebra $A_{K}^{\text {arith }}$. More precisely we will construct a (commutative) diagram (see Section 6.3)

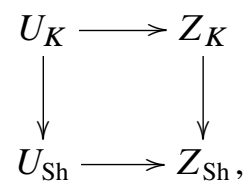

which is induced by a morphism of Shimura data $\delta_{K} \rightarrow \oint_{\mathrm{Sh}}$ constructed in Section 3.3.

Then, using the Criterion 5.1, we see that the morphism $Z_{\mathrm{Sh}}^{+} \rightarrow Z_{\mathrm{Sh}}$ (from the above diagram) is invertible and obtain in this way a continuous map

$$
\Theta: Z_{K} \rightarrow Z_{\mathrm{Sh}} \rightarrow Z_{\mathrm{Sh}}^{+} \rightarrow Z_{\mathrm{Sh}}^{\mathrm{ad}} .
$$

Using easy properties of the automorphism group of $\mathcal{M}^{\mathrm{cm}}$, we can model each $f$ in $\mathcal{M}^{\mathrm{cm}}$ as a function $\tilde{f}$ on the space $Z_{\mathrm{Sh}}^{\text {ad }}$ (which might have singularities). Nevertheless in Proposition 7.1 we see that for every $f \in \mathcal{M}^{\mathrm{cm}}$ the pull back $\tilde{f} \circ \Theta$ lies in $H_{K}=C_{c}\left(Z_{K}\right)$ and we can define $A_{K}^{\text {arith }}$ as the $K$-algebra generated by these elements, i.e.,

$$
A_{K}^{\text {arith }}=\left\langle\tilde{f} \circ \Theta \mid f \in \mathcal{M}^{\mathrm{cm}}\right\rangle_{K} .
$$

Now, using the classification of extremal $\mathrm{KMS}_{\infty}$-states of $\mathcal{A}_{K}$ (see Section 6.1.5), the verification of property (v) is an immediate consequence of our construction and property (vi) follows by using Shimura's reciprocity law and the observation made in Proposition 4.4 (see Section 7.1 for the details). 
Our paper is organized along the lines of this section, recalling on the way the necessary background. In addition we put some effort in writing a long Appendix which covers hopefully enough information to make this paper "readable" for a person with little beforehand knowledge of the arithmetic theory of Shimura varieties.

Acknowledgment. The author would like to thank James Milne, Sergey Neshveyev and Frederic Paugam for helpful and valuable comments and his advisor Eric Leichtnam for his guidance through this first project of the author's $\mathrm{PhD}$ thesis.

This work has been supported by the Marie Curie Research Training Network MRTN-CT-2006-031962 in Noncommutative Geometry, EU-NCG.

Notations and conventions. We use the common notations $\mathbb{N}, \mathbb{Z}, \mathbb{Q}, \mathbb{R}, \mathbb{C}$. If $A$ denotes a ring or monoid, we denote its group of multiplicative units by $A^{\times}$. A number field is a finite extension of $\mathbb{Q}$. The ring of integers of a number field $K$ is denoted by $\mathcal{O}_{K}$. We denote by $\mathbb{A}_{K}=\mathbb{A}_{K, f} \times \mathbb{A}_{K, \infty}$ the adele ring of $K$ (with its usual topology), where $\mathbb{A}_{K, f}$ denotes the finite adeles and $\mathbb{A}_{K, \infty}$ the infinite adeles of $K$. $\mathbb{A}_{K}$ contains $K$ by the usual diagonal embedding and by $\hat{\mathcal{O}}_{K}$ we denote the closure of $\mathcal{O}_{K}$ in $\mathbb{A}_{K, f}$. Invertible adeles are called ideles. The idele class group $\mathbb{A}_{K}^{\times} / K^{\times}$of $K$ is denoted by $C_{K}$, its connected component of the identity by $D_{K}$.

We fix an algebraic closure $\overline{\mathbb{Q}}$ of $\mathbb{Q}$ in $\mathbb{C}$. Usually we think of a number field $K$ as lying in $\mathbb{C}$ by an embedding $\tau: K \rightarrow \overline{\mathbb{Q}} \subset \mathbb{C}$. Complex conjugation on $\mathbb{C}$ is denoted by $\iota$. Sometimes we write $z^{\iota}$ for the complex conjugate of a complex number $z$.

Artin's reciprocity map $\mathbb{A}_{K}^{\times} \rightarrow \operatorname{Gal}\left(K^{\mathrm{ab}} / K\right): v \mapsto[v]$ is normalized such that an uniformizing parameter maps to the arithmetic Frobenius element. Further given a group $G$ acting partially on a set $X$ we denote by

$$
G \boxplus X=\{(g, x) \in G \times X \mid g x \in X\}
$$

the corresponding groupoid (see [LLN09], p. 327).

If $X$ denotes a topological space we write $\pi_{0}(X)$ for its set of connected components.

\section{On the arithmetic subalgebra for $K=\mathbb{Q}(i)$}

Before we describe our general construction we will explain the easiest case $K=$ $\mathbb{Q}(i)$, where many simplifications occur, in some detail and point out the modifications necessary for the general case. For the remainder of this section $K$ always denotes $\mathbb{Q}(i)$, although many of the definitions work in general. For the convenience of the reader we will try to make the following section as self-contained as possible.

2.1. The quotient map $U_{K} \rightarrow Z_{K}$. We denote by $T^{K}$ the $\mathbb{Q}$-algebraic torus given by the Weil restriction $T^{K}=\operatorname{Res}_{K / \mathbb{Q}}\left(\mathbb{G}_{m, K}\right)$ of the multiplicative group $\mathbb{G}_{m, K}$, i.e., for a $\mathbb{Q}$-algebra $R$ the $R$-points of $T^{K}$ are given by $T^{K}(R)=(R \otimes \mathbb{Q} K)^{\times}$. In 
particular we see that $T^{K}(\mathbb{Q})=K^{\times}, T^{K}\left(\mathbb{A}_{f}\right)=\mathbb{A}_{K, f}^{\times}$and $T^{K}(\mathbb{R})=\mathbb{A}_{K, \infty}^{\times}$. In our special case we obtain that after extending scalars to $\mathbb{R}$ the $\mathbb{R}$-algebraic group $T_{\mathbb{R}}^{K}$ is isomorphic to $\mathbb{S}=\operatorname{Res}_{\mathbb{C} / \mathbb{R}}\left(\mathbb{G}_{m, \mathbb{R}}\right)$. Further the finite set $X_{K}=T^{K}(\mathbb{R}) / T^{K}(\mathbb{R})^{+}=$ $\pi_{0}\left(T^{K}(\mathbb{R})\right)$ consists in our case of only one point. With this in mind we consider the 0-dimensional Shimura datum (see D.5)

$$
\varsigma_{K}=\left(T^{K}, X_{K}, h_{K}\right),
$$

where the morphism $h_{K}: \$ \rightarrow T_{\mathbb{R}}^{K}$ is simply given by the identity (due to $T_{\mathbb{R}}^{K} \cong \$$ ). (In the general case $h_{K}$ is chosen according to Lemma 3.1.)

The (0-dimensional) Shimura variety $\operatorname{Sh}\left(\delta_{K}\right)$ is in our case of the simple form

$$
\operatorname{Sh}\left(S_{K}\right)=T^{K}(\mathbb{Q}) \backslash\left(X_{K} \times T^{K}\left(\mathbb{A}_{f}\right)\right)=K^{\times} \backslash \mathbb{A}_{K, f}^{\times} .
$$

We write $[z, l]$ for an element in $\operatorname{Sh}\left(S_{K}\right)$ meaning that $z \in X_{K}$ and $l \in T^{K}\left(\mathbb{A}_{f}\right)$.

(For general number fields the description of $\operatorname{Sh}\left(\delta_{K}\right)$ is less explicit but no difficulty occurs.)

Remark 1. The reader should notice that by class field theory we can identify $\operatorname{Sh}\left(S_{K}\right)=K^{\times} \backslash \mathbb{A}_{K, f}^{\times}$with the Galois $\operatorname{group} \operatorname{Gal}\left(K^{\mathrm{ab}} / K\right)$ of the maximal abelian extension $K^{\mathrm{ab}}$ of $K$. This is true in general, see Section 6.1.4.

The (topological) groupoid $U_{K}$ underlying the BC-type system

$$
\mathcal{A}_{K}=\left(A_{K},\left(\sigma_{t}\right)_{t \in \mathbb{R}}\right)
$$

is now of the form (see (4) for the notation)

$$
U_{K}=T^{K}\left(\mathbb{A}_{f}\right) \boxplus\left(\hat{\mathcal{O}}_{K} \times \operatorname{Sh}\left(S_{K}\right)\right)
$$

with the natural action of $T^{K}\left(\mathbb{A}_{f}\right)$ on $\operatorname{Sh}\left(S_{K}\right)$ (see D.2) and the partial action of $T^{K}\left(\mathbb{A}_{f}\right)=\mathbb{A}_{K, f}^{\times}$on the multiplicative semigroup $\hat{\mathcal{O}}_{K} \subset \mathbb{A}_{K, f}$ by multiplication. The group

$$
\Gamma_{K}^{2}=\hat{\mathcal{O}}_{K}^{\times} \times \hat{\mathcal{O}}_{K}^{\times}
$$

is acting on $U_{K}$ as

$$
\left(\gamma_{1}, \gamma_{2}\right)(g, \rho,[z, l])=\left(\gamma_{1}^{-1} g \gamma_{2}, \gamma_{2} \rho,\left[z, l \gamma_{2}^{-1}\right]\right),
$$

where $\gamma_{1}, \gamma_{2} \in \widehat{\mathcal{O}}_{K}^{\times}, g, l \in T^{K}\left(\mathbb{A}_{f}\right), \rho \in \widehat{\mathcal{O}}_{K}$ and $z \in X_{K}$, and we obtain the quotient map

$$
U_{K} \rightarrow Z_{K}=\Gamma_{K}^{2} \backslash U_{K}
$$

In the end of this section we will construct the arithmetic subalgebra $A_{K}^{\text {arith }}$ of the BC-type system $\mathcal{A}_{K}$ which is contained in $H_{K}=C_{c}\left(Z_{K}\right) \subset A_{K}$. For this we will need the following. 
2.2. The quotient map $\boldsymbol{U}_{\mathrm{Sh}} \rightarrow \boldsymbol{Z}_{\mathrm{Sh}}$. In our case of $K=\mathbb{Q}(i)$ the maximal CM subfield $E$ of $K$ is equal to $K$. The Shimura datum $\delta_{\mathrm{Sh}}$ associated with $E$ is of the form (see Section 3.2)

$$
\oint_{\mathrm{Sh}}=\left(\operatorname{GSp}\left(V_{E}, \psi_{E}\right), \mathbb{\boxplus}^{ \pm}, h_{\mathrm{cm}}\right) .
$$

Here $\mathrm{GSp}=\mathrm{GSp}\left(V_{E}, \psi_{E}\right)$ is the general symplectic group (cf. D.3) associated with the symplectic vector space $\left(V_{E}, \psi_{E}\right)$.

The latter is in general chosen according to (13). Due to the fact that the reflex field $E^{*}$ (cf. B.3) is equal to $E$ and the Serre group $S^{E}$ is equal to $T^{E}=T^{K}$ we can simply choose the $\mathbb{Q}$-vector space $V_{E}$ to be the $\mathbb{Q}$-vector space $E$ and the symplectic form $\psi_{E}: E \times E \rightarrow \mathbb{Q}$ to be the map $(x, y) \mapsto \operatorname{Tr}_{E / \mathbb{Q}}\left(i x y^{l}\right)$. A simple calculation shows that $\psi_{E}(f(x), f(y))=\operatorname{det}(f) \psi_{E}(x, y)$ for all $f \in \operatorname{End}_{\mathbb{Q}}\left(V_{E}\right)$ and all $x, y \in V_{E}$, therefore we can identify GSp with $\mathrm{GL}_{2}=\mathrm{GL}\left(V_{E}\right)$. Now again using the fact that the Serre group $S^{E}$ equals $T^{E}$ we see that the general construction of $h_{\mathrm{cm}}: \mathbb{S}=T_{\mathbb{R}}^{E} \rightarrow \mathrm{GSp}_{\mathbb{R}}=\mathrm{GL}_{2, \mathbb{R}}$ (see (16)) is given on the $\mathbb{R}$-points by $a+i b \in \mathbb{C}^{\times}=\mathbb{S}(\mathbb{R}) \rightarrow\left(\begin{array}{cc}a & -b \\ b & a\end{array}\right) \in \mathrm{GL}_{2}(\mathbb{R})$. Each $\alpha \in \mathrm{GSp}(\mathbb{R})$ defines a map $\alpha^{-1} h_{\mathrm{cm}} \alpha: \mathbb{S} \rightarrow \mathrm{GSp}_{\mathbb{R}}$ given on the $\mathbb{R}$-points by $a+i b \in \mathbb{C}^{\times} \mapsto \alpha^{-1} h_{\mathrm{cm}}(a+i b) \alpha \in$ $\mathrm{GL}_{2}(\mathbb{R})$ and the GSp $(\mathbb{R})$-conjugacy class $X=\left\{\alpha^{-1} h_{\mathrm{cm}} \alpha \mid \alpha \in \mathrm{GSp}_{\mathbb{R}}(\mathbb{R})\right\}$ of $h_{\mathrm{cm}}$ can be identified with the Siegel upper lower half space $\mathbb{U}^{ \pm}=\mathbb{C}-\mathbb{R}$ by the map

$$
\alpha^{-1} h_{\mathrm{cm}} \alpha \in X \mapsto\left(\alpha^{-1} h_{\mathrm{cm}}(i) \alpha\right) \cdot i \in \mathbb{U}^{ \pm},
$$

where the latter action - denotes Möbius transformation. Under this identification the morphism $h_{\mathrm{cm}}$ corresponds to the point $x_{\mathrm{cm}}=i$ on the upper half plane $\mathbb{H}$. The point $x_{\mathrm{cm}} \in \mathbb{U}$ is a so-called CM-point (see D.6).

Remark. The definition of a CM-point and the observation made in (15) explain the need of using the Serre group in the general construction of $h_{\mathrm{cm}}$. The explanation given in Section 4.1 shows in particular why we have to define the vector space $V_{E}$ in general according to (13).

The Shimura variety $\operatorname{Sh}\left(\delta_{\mathrm{Sh}}\right)$ is of the nice form (cf. (39))

$$
\operatorname{Sh}\left(S_{\mathrm{Sh}}\right)=\operatorname{GSp}(\mathbb{Q}) \backslash\left(\mathbb{Q}^{ \pm} \times \operatorname{GSp}\left(\mathbb{A}_{f}\right)\right) .
$$

Again we write elements as $[z, l] \in \operatorname{Sh}\left(\mathcal{S}_{\mathrm{Sh}}\right)$ with $z \in \mathbb{M}^{ \pm}$and $g \in \operatorname{GSp}\left(\mathbb{A}_{f}\right)$.

In our case the topological groupoid $U_{\mathrm{Sh}}$ underlying the Shimura system $\mathcal{A}_{\mathrm{Sh}}$ is given by (cf. Section 6.2.1)

$$
U_{\mathrm{Sh}}=\operatorname{GSp}\left(\mathbb{A}_{f}\right) \boxplus\left(M_{2}(\widehat{\mathbb{Z}}) \times \operatorname{Sh}\left(\delta_{\mathrm{Sh}}\right)\right),
$$

where $\operatorname{GSp}\left(\mathbb{A}_{f}\right)=\mathrm{GL}_{2}\left(\mathbb{A}_{f}\right)$ is acting in the natural way on $\operatorname{Sh}\left(\delta_{\mathrm{Sh}}\right)$ and partially on the multiplicative monoid of $(2 \times 2)$-matrices $M_{2}(\widehat{\mathbb{Z}}) \subset M_{2}\left(\mathbb{A}_{f}\right)$ with entries in $\hat{\mathbb{Z}}$. The group

$$
\Gamma_{\mathrm{Sh}}^{2}=\mathrm{GL}_{2}(\hat{\mathbb{Z}}) \times \mathrm{GL}_{2}(\widehat{\mathbb{Z}})
$$


is acting on $U_{\text {Sh }}$ exactly like in (5) and induces the quotient map

$$
U_{\mathrm{Sh}} \rightarrow Z_{\mathrm{Sh}}=\Gamma_{\mathrm{Sh}}^{2} \backslash U_{\mathrm{Sh}}
$$

Remark. Note that the quotient $Z_{\mathrm{Sh}}$ is not a groupoid anymore (see [HP05], top of p. 251).

In our example it is sufficient to consider the positive groupoid $U_{\mathrm{Sh}}^{+}$(see 6.2.2) associated with $U_{\mathrm{Sh}}$. (In the general case the adjoint groupoid $U_{\mathrm{Sh}}^{\text {ad }}$ seems to be more appropriate.) It is given by

$$
U_{\mathrm{Sh}}^{+}=\mathrm{GSp}(\mathbb{Q})^{+} \boxplus\left(M_{2}(\widehat{\mathbb{Z}}) \times \mathbb{U}\right)
$$

together with the group

$$
\left(\Gamma_{\mathrm{Sh}}^{+}\right)^{2}=\mathrm{GL}_{2}(\mathbb{Z})^{+} \times \mathrm{GL}_{2}(\mathbb{Z})^{+}=\mathrm{SL}_{2}(\mathbb{Z}) \times \mathrm{SL}_{2}(\mathbb{Z})
$$

acting by

$$
\left(\gamma_{1}, \gamma_{2}\right)(g, \rho, z)=\left(\gamma_{1} g \gamma_{2}^{-1}, \gamma_{2} \rho, \gamma_{2} z\right)
$$

and inducing the quotient map

$$
U_{\mathrm{Sh}}^{+} \rightarrow Z_{\mathrm{Sh}}^{+}=\left(\Gamma_{\mathrm{Sh}}^{+}\right)^{2} \backslash U_{\mathrm{Sh}}^{+} .
$$

By construction $\operatorname{GSp}(\mathbb{R})$ is acting (free and transitively) on $\mathbb{M}^{ \pm}$and $\operatorname{GSp}(\mathbb{R})^{+}$, the connected component of the identity, can be thought of as stabilizer of the upper half plane $\mathbb{H}^{+}=\mathbb{W}$ which explains the action of $\operatorname{GSp}(\mathbb{Q})^{+}=\operatorname{GSp}(\mathbb{Q}) \cap \mathrm{GSp}(\mathbb{R})^{+}$on $\mathbb{H}$. Due to Criterion 5.1 we know that the natural (equivariant) morphism of topological groupoids $U_{\mathrm{Sh}}^{+} \rightarrow U_{\mathrm{Sh}}$ given by $(g, \rho, z) \mapsto(g, \rho,[z, 1])$ induces a homeomorphism on the quotient spaces $Z_{\mathrm{Sh}}^{+} \longrightarrow Z_{\mathrm{Sh}}$ in the commutative diagram

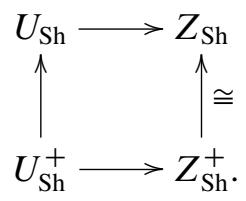

Remark. The groupoid $U_{\mathrm{Sh}}^{+}$corresponds to the $\mathrm{GL}_{2}$-system of Connes and Marcolli (see [CM08] and [HP05], Lemma 5.8).

2.3. A map relating $\boldsymbol{U}_{\boldsymbol{K}}$ and $\boldsymbol{U}_{\mathrm{Sh}}$. We want to define an equivariant morphism of topological groupoids $U_{K} \rightarrow U_{\mathrm{Sh}}$, where equivariance is meant with respect to the actions of $\Gamma_{K}$ on $U_{K}$ and $\Gamma_{\text {Sh }}$ on $U_{\text {Sh. }}$. For this it is necessary (and more or less sufficient) to construct a morphism of Shimura data between $S_{K}=\left(T^{K}, X_{K}, h_{K}\right)$ and $\left.S_{\mathrm{Sh}}=\left(\operatorname{GSp}\left(V_{E}, \psi_{E}\right)\right), \mathbb{U}^{ \pm}, h_{\mathrm{cm}}\right)$, which is given by a morphism of algebraic groups

$$
\varphi: T^{K} \rightarrow \mathrm{GSp}
$$


such that $h_{\mathrm{cm}}=\varphi_{\mathbb{R}} \circ h_{K}$. In our case the general construction of $\varphi$, stated in (17), reduces to the simple map (on the $\mathbb{Q}$-points)

$$
a+i b \in K^{\times}=T^{K}(\mathbb{Q}) \mapsto\left(\begin{array}{rr}
a & -b \\
b & a
\end{array}\right) \in \mathrm{GL}_{2}(\mathbb{Q})=\mathrm{GSp}(\mathbb{Q}),
$$

and we see in fact that after extending scalars to $\mathbb{R}$ the morphism $\varphi_{\mathbb{R}}: T_{\mathbb{R}}^{K}=\mathbb{\$} \rightarrow$ $\mathrm{GSp}_{\mathbb{R}}$ is already equal to $h_{\mathrm{cm}}: \mathbb{S} \rightarrow \mathrm{GSp}_{\mathbb{R}}$. The simplicity of our example comes again from the fact that we do not have to bother about the Serre group, which makes things less explicit, although the map $\varphi: T^{K} \rightarrow$ GSp still has a quite explicit description even in the general case thanks to Lemma 3.3.

Now by functoriality (see D.5) we obtain a morphism of Shimura varieties

$$
\operatorname{Sh}(\varphi): \operatorname{Sh}\left(\delta_{K}\right) \rightarrow \operatorname{Sh}\left(\delta_{\mathrm{Sh}}\right)
$$

which can be explicitly described by

$$
[z, l] \in K^{\times} \backslash\left(X_{K} \times T^{K}\left(\mathbb{A}_{f}\right)\right) \mapsto\left[x_{\mathrm{cm}}, \varphi\left(\mathbb{A}_{f}\right)(l)\right] \in \mathrm{GSp}(\mathbb{Q}) \backslash\left(\mathbb{\boxplus}^{ \pm} \times \mathrm{GSp}\left(\mathbb{A}_{f}\right)\right) .
$$

In the general case we have essentially the same description (see (30)), the point being that every element $z$ in $X_{K}$ is mapped to $x_{\mathrm{cm}} \in \mathbb{W}^{ \pm}$, as in the general case. Using $\hat{\mathcal{O}}_{K}=\widehat{\mathbb{Z}} \otimes_{\mathbb{Z}} \mathcal{O}_{K}$ we can continue the map $\varphi\left(\mathbb{A}_{f}\right)$ to a morphism of (topological) semigroups $M(\varphi)\left(\mathbb{A}_{f}\right): \widehat{\mathcal{O}}_{K} \rightarrow M_{2}(\widehat{\mathbb{Z}})$ by setting $n \otimes(a+i b) \mapsto\left(\begin{array}{cc}a n & -b n \\ b n & a n\end{array}\right)$. By continuation we mean that $\varphi\left(\mathbb{A}_{f}\right)$ and $M(\varphi)\left(\mathbb{A}_{f}\right)$ agree on the intersection of $T^{K}\left(\mathbb{A}_{f}\right) \cap \hat{\mathcal{O}}_{K} \subset \mathbb{A}_{K, f}$. In the general case the explicit description of $\varphi$ given in (18) is used to continue $\varphi$ to $M(\varphi)$ (see Section 6.3.1), the above example being a special case. Now it can easily be checked that

$$
(g, \rho,[z, l]) \in U_{K} \mapsto\left(\varphi\left(\mathbb{A}_{f}\right)(g), M(\varphi)\left(\mathbb{A}_{f}\right)(\rho),\left[x_{\mathrm{cm}}, \varphi\left(\mathbb{A}_{f}\right)(l)\right]\right) \in U_{\mathrm{Sh}}
$$

defines the desired equivariant morphism of topological groupoids

$$
U_{K} \rightarrow U_{\mathrm{Sh}} .
$$

Summarizing we obtain the commutative diagram (using (7))

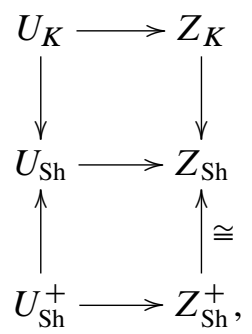

which gives us the desired morphism of topological spaces

$$
\Theta: Z_{K} \rightarrow Z_{\mathrm{Sh}} \rightarrow Z_{\mathrm{Sh}}^{+} .
$$


In general we have to go one step further and use the adjoint groupoid $Z_{\mathrm{Sh}}^{\text {ad }}$ (cf. Section 6.2.2), but this is only due to the general description of the automorphism group $\operatorname{Aut}_{\mathbb{Q}}(\mathcal{M})$ of the field of arithmetic automorphic functions $\mathcal{M}$; see Section 4.3.1 and the next section for explanations.

2.4. Interlude: theory of complex multiplication. In this section we will provide the number theoretic background which is necessary to understand the constructions done so far.

We are interested in constructing the maximal abelian extension $E^{\mathrm{ab}}$ of $E=K=$ $\mathbb{Q}(i)$ and there are in general two known approaches to this problem.

I) The elliptic curve $A$ : $y^{2}=x^{3}+x$. Let us denote by $A$ the elliptic curve defined by the equation

$$
A: y^{2}=x^{3}+x .
$$

It is known that the field of definition of $A$ and its torsion points generate the maximal abelian extension $E^{\mathrm{ab}}$ of $E$. (By field of definition of the torsion points of $A$ we mean the coordinates of the torsion points.)

Notice that in our case the complex points of our elliptic curve $A$ are given by $A(\mathbb{C})=\mathbb{C} / \mathcal{O}_{E}$ and the rational ring of endomorphisms of $A$ turns out to be $\operatorname{End}(A(\mathbb{C}))_{\mathbb{Q}}=\mathbb{Q} \otimes_{\mathbb{Z}} \mathcal{O}_{E}=E$; we say that $A$ has complex multiplication by $E$. Remember that $\mathcal{O}_{E}=\mathbb{Z}[i]$.

Remark. To obtain the abelian extensions of $K$ provided by $A$ explicitly one may use for example the Weierstrass p-function associated with $A$ (see [Sil94]) but as we will use another approach we do not want to dive into this beautiful part of explicit class field theory.

II) The (Siegel) Modular curve $\operatorname{Sh}\left(\mathbf{G S p}, \mathbb{Q}^{ \pm}, \boldsymbol{h}_{\mathrm{cm}}\right)$. We want to interpret the Shimura variety $\mathrm{Sh}\left(\oint_{\mathrm{Sh}}\right)=\mathrm{Sh}\left(\mathrm{GSp}, \mathbb{R}^{ \pm}, h_{\mathrm{cm}}\right)$ constructed in the last section as moduli space of elliptic curves with torsion data.

The moduli theoretic picture. For this we consider the connected component $\mathrm{Sh}^{o}=$ $\operatorname{Sh}\left(\delta_{\mathrm{Sh}}\right)^{o}$ of our Shimura variety which is described by the projective system (see D.4 and [Mil04])

$$
\mathrm{Sh}^{o}=\lim _{N} \Gamma(N) \backslash \mathbb{W},
$$

where $\Gamma(N)$, for $N \geq 1$, denotes the subgroup of $\Gamma=\Gamma(1)=\mathrm{SL}_{2}(\mathbb{Z})$ defined by $\Gamma(N)=\left\{g \in \Gamma \mid g \equiv\left(\begin{array}{ll}1 & 0 \\ 0 & 1\end{array}\right) \bmod N\right\}$. We can view the quotient $\Gamma(N) \backslash \mathbb{W}$ as a complex analytic space, but due to the work of Baily and Borel it carries also a unique structure of an algebraic variety over $\mathbb{C}$ (see [Mil04]). We will use both viewpoints. Seen as an analytic space we write $\mathbb{U}(N)=\Gamma(N) \backslash \mathbb{W}$ and for the algebraic space we write $\mathrm{Sh}_{N}^{o}=\Gamma(N) \backslash \mathbb{R}$. 
Now observe that the space $\mathbb{U}(N)$ classifies isomorphism classes of pairs $(A, t)$ given by an elliptic curve $A$ over $\mathbb{C}$ together with a $N$-torsion point $t$ of $A$. In particular $\mathbb{U}(1)=\Gamma \backslash \mathbb{W}$ classifies isomorphism classes of elliptic curves over $\mathbb{C}$.

In this picture our CM-point $\left[x_{\mathrm{cm}}\right]_{1}=[i]_{1} \in \mathbb{U}(1)$ corresponds to the isomorphism class of the elliptic curve $A$ from (9) and, more general, the points $\left[x_{\mathrm{cm}}\right]_{N} \in$ $\mathbb{U}(N)$ capture the field of definition of $A$ and its various torsion points and recover the maximal abelian extension $E^{\mathrm{ab}}$ of $E$ in this way! By $[z]_{N}$ we denote the image of $z \in \mathbb{U}$ in $\mathbb{U}(N)$ under the natural quotient map $\mathbb{U} \rightarrow \mathbb{U}(N)$.

Remark. For the relation between $\mathrm{Sh}$ and $\mathrm{Sh}^{o}$ we refer the reader to [Mil04], p. $303 \mathrm{ff}$.

The field of arithmetic modular functions $\mathcal{M}$. To construct the abelian extensions provided by the various points $\left[x_{\mathrm{cm}}\right]_{N}$ explicitly we proceed as follows: We consider the connected canonical model $M^{o}$ of $\mathrm{Sh}^{o}$ (see D.7) which provides us with an algebraic model $M_{N}^{o}=\Gamma(N) \backslash M^{o}$ of the algebraic variety $\mathrm{Sh}_{N}^{o}$ over the cyclotomic field $\mathbb{Q}\left(\zeta_{N}\right)$. In general we obtain algebraic models over subfields of $\mathbb{Q}^{\mathrm{ab}}$. This means that $M_{N}^{o}$ is an algebraic variety defined over the cyclotomic field $\mathbb{Q}\left(\zeta_{N}\right)$ and after scalar extension to $\mathbb{C}$ it becomes isomorphic to the complex algebraic variety $\mathrm{Sh}_{N}^{o}$. Let us denote by $k\left(M_{N}^{o}\right)$ the field of rational functions on $M_{N}^{o}$, in particular this means that elements in $k\left(M_{N}^{o}\right)$ are rational over $\mathbb{Q}\left(\zeta_{N}\right)$. It makes sense to view the point $\left[x_{\mathrm{cm}}\right]_{N}$ as a point on $M_{N}^{o}$ and if a function $f \in k\left(M_{N}^{o}\right)$ is defined at $\left[x_{\mathrm{cm}}\right]_{N}$, then we know (cf. Section 4.3.1) that

$$
f\left(\left[x_{\mathrm{cm}}\right]_{N}\right) \in E^{\mathrm{ab}} .
$$

In particular varying over the various $N$ and the rational functions in $k\left(M_{N}^{o}\right)$ the values $f\left(\left[x_{\mathrm{cm}}\right]_{N}\right)$ generate $E^{\mathrm{ab}}$ over $E$. The next step is to realize that the function field $k\left(M_{N}^{o}\right)$ can be seen as subset of the field of rational functions $k\left(\mathrm{Sh}_{N}^{o}\right)$ on the complex algebraic variety $\mathrm{Sh}_{N}^{o}$ (cf. Section 4.3.1). As rational functions in $k\left(\mathrm{Sh}_{N}^{o}\right)$ correspond to meromorphic functions on $\mathbb{U}(N)$ and meromorphic functions on $\mathbb{U}(N)$ are nothing else than meromorphic functions on $\mathbb{Q} \mathbb{W}$ that are invariant under the action of $\Gamma(N)$, we can view each rational function in $k\left(M_{N}^{o}\right)$ as a meromorphic function on $\mathbb{t}$ which is invariant under $\Gamma(N)$. If we denote by $k\left(M_{N}^{o}\right)_{\text {cusp }}$ the subfield of $k\left(M_{N}^{o}\right)$ consisting of functions $f \in k\left(M_{N}^{o}\right)$ that give rise to meromorphic functions on $\mathbb{W}$ that are meromorphic at the cusps (see Section 4.3.1), it makes sense to define the field of meromorphic functions $\mathcal{M}$ on $\mathbb{U}$ given by the union

$$
\mathcal{M}=\bigcup_{N} k\left(M_{N}^{o}\right)_{\text {cusp }} \text {. }
$$

Due to (10) we know furthermore that for every $f \in \mathcal{M}$ which is defined in $x_{\mathrm{cm}}$, we have

$$
f\left(x_{\mathrm{cm}}\right) \in K^{\mathrm{ab}}=E^{\mathrm{ab}}
$$

and $K^{\mathrm{ab}}$ is generated in this way. Therefore we call the field $\mathcal{M}$ the field of arithmetic modular functions. 
Explicitly $\mathcal{M}$ is described for example in [Shi00] or [CM08], Definition 3.60, p. 507. A very famous arithmetic modular function is given by the $j$-function which generates the Hilbert class field of an arbitrary imaginary quadratic field.

Remark. (1) In the light of Section 4.1 and Lemma 3.2 (2) we mention that the field of definition $E\left(x_{\mathrm{cm}}\right)$ of $x_{\mathrm{cm}}$ is in our example equal to $E=\mathbb{Q}(i)$, which is the reason why our example is especially simple.

(2) If we take a generic meromorphic function $g$ on $\mathbb{U}(N)$ that is defined in $\left[x_{\mathrm{cm}}\right]_{N}$ then the value $g\left(\left[x_{\mathrm{cm}}\right]_{N}\right) \in \mathbb{C}$ will not even be algebraic. This is the reason why we need the canonical model $M^{o}$ which provides an arithmetic structure for the field of meromorphic functions on $\mathbb{U}(N)$.

In the general case the construction of $\mathcal{M}$ is quite similar to the construction above (see Section 4), the only main difference being that in general the theory is much less explicit (e.g. the description of $\mathcal{M}$ ).

Automorphisms of $\mathcal{M}$ and Shimura's reciprocity law. In our example $K=E=$ $\mathbb{Q}(i)$, using the notation from Section 4.3 , we have the equality $\overline{\mathcal{E}}=\frac{\mathrm{GSp}\left(\mathbb{A}_{f}\right)}{\mathbb{Q}^{\times}}$and obtain a group homomorphism

$$
\operatorname{GSp}\left(\mathbb{A}_{f}\right) \rightarrow \overline{\mathcal{E}} \rightarrow \operatorname{Aut}_{\mathbb{Q}}(\mathcal{M}),
$$

where the first arrow is simply the projection and the second arrow comes from Section 4.3.2. We denote the action of $\alpha \in \operatorname{GSp}\left(\mathbb{A}_{f}\right)$ on a function $f \in \mathcal{M}$ by ${ }^{\alpha} f$. In particular we see that $\alpha \in \mathrm{GSp}(\mathbb{Q})^{+}=\mathrm{SL}_{2}(\mathbb{Q})$ is acting by

$$
{ }^{\alpha} f=f \circ \alpha^{-1},
$$

where $\alpha^{-1}$ acts on $\mathbb{W}$ by Möbius transformation. The adjoint system, which is well suited for the general case (cf. Section 6.2.2), is not needed in our special example. We note that the group $\mathrm{GSp}^{\text {ad }}(\mathbb{Q})^{+}$occurring in Section 6.2.2 is given by $\mathrm{SL}_{2}(\mathbb{Q}) /\{ \pm I\}$, where $I$ denotes the unit in $\mathrm{SL}_{2}(\mathbb{Q})$. Due to the fact that $\{ \pm I\}$ acts trivially on $\mathbb{H}$ we can lift the action to $\operatorname{GSp}(\mathbb{Q})^{+}$. Further there is a morphism of algebraic groups

$$
\eta: T^{K} \rightarrow \mathrm{GSp}
$$

which induces a group homomorphism denoted by (compare (22))

$$
\bar{\eta}=\eta\left(\mathbb{A}_{f}\right): T^{K}\left(\mathbb{A}_{f}\right) \rightarrow \operatorname{GSp}\left(\mathbb{A}_{f}\right) .
$$

The reciprocity law of Shimura can be stated in our special case as follows:

Let $v$ be in $\mathbb{A}_{K, f}^{\times}$, denote by $[v] \in \operatorname{Gal}\left(K^{\mathrm{ab}} / K\right)$ its image under Artin's reciprocity map and let $f \in \mathcal{M}$ be defined in $x_{\mathrm{cm}} \in \mathbb{H}$. Then ${ }^{\bar{\eta}(v)} f$ is also defined in $x_{\mathrm{cm}} \in \mathbb{W}$ and

$$
\bar{\eta}^{(v)} f\left(x_{\mathrm{cm}}\right)=[v]^{-1} f\left(x_{\mathrm{cm}}\right) \in K^{\mathrm{ab}} .
$$

The formulation in the general case concentrates on the group $\overline{\mathcal{E}}$ (see Section 4.4).

Now we are ready for the construction of the arithmetic subalgebra. 
2.5. Construction of the arithmetic subalgebra. Define $\mathcal{M}^{\mathrm{cm}}$ to be the subring of functions in $\mathcal{M}$ which are defined at $x_{\mathrm{cm}} \in \mathbb{H}$. For every $f \in \mathcal{M}^{\mathrm{cm}}$ we define a function $\tilde{f}$ on the groupoid $U_{\mathrm{Sh}}^{+}$by

$$
\tilde{f}(g, \rho, z)= \begin{cases}\rho^{\rho} f(z) & \text { if } \rho \in \mathrm{GSp}(\widehat{\mathbb{Z}}), \\ 0 & \text { if } \rho \in M_{2}(\widehat{\mathbb{Z}})-\mathrm{GSp}(\widehat{\mathbb{Z}}) .\end{cases}
$$

Due to (11) $\tilde{f}$ is invariant under the action of $\Gamma_{\mathrm{Sh}}^{+}=\mathrm{SL}_{2}(\mathbb{Z}) \times \mathrm{SL}_{2}(\mathbb{Z})$ and therefore $\tilde{f}$ descends to the quotient $Z_{\mathrm{Sh}}^{+}$(cf. (6)). Proposition 7.1 shows further that the pullback $\tilde{f} \circ \Theta$ (see (8)) defines a compactly supported, continuous function on $Z_{K}$, i.e., we have $\tilde{f} \circ \Theta \in H_{K}=C_{c}\left(Z_{K}\right) \subset A_{K}$. Therefore we can define the $K$-subalgebra $A_{K}^{\text {arith }}$ of $H_{K}$ generated by these elements

$$
A_{K}^{\text {arith }}=\left\langle\tilde{f} \circ \Theta \mid f \in \mathcal{M}^{\mathrm{cm}}\right\rangle_{K} .
$$

Now we want to show that $A_{K}^{\text {arith }}$ is indeed an arithmetic subalgebra for $\mathcal{A}_{K}$ (see (1)).

The set $\varepsilon_{\infty}$ of extremal $\mathrm{KMS}_{\infty}$-states of $\mathcal{A}_{K}$ is indexed by the set $\mathrm{Sh}\left(S_{K}\right)$ and for every $\omega \in \operatorname{Sh}\left(\delta_{K}\right)$ the corresponding $\mathrm{KMS}_{\infty}$-state $\varrho_{\omega}$ is given on an element $f \in H_{K}=C_{c}\left(Z_{K}\right)$ by (cf. Section 6.1.5)

$$
\varrho_{\omega}(f)=f(1,1, \omega) .
$$

Using Remark 1 we write $[\omega]$ for an element $\omega \in \operatorname{Sh}\left(\delta_{K}\right)$ when regarded as element in $\operatorname{Gal}\left(K^{\mathrm{ab}} / K\right)$. Now if we take a function $f \in \mathcal{M}^{\mathrm{cm}}$ and $\omega \in \operatorname{Sh}\left(\mathcal{S}_{K}\right)$ we immediately see

$$
\varrho_{\omega}(\tilde{f} \circ \Theta)=[\omega]^{-1}\left(f\left(x_{\mathrm{cm}}\right)\right) \in K^{\mathrm{ab}}
$$

and property (v) follows (in general we will only show property (v) of course). To show property (vi) we take a symmetry $v \in C_{K}=\mathbb{A}_{K}^{\times} / K^{\times}$(see Section 6.1.4) of $\mathcal{A}_{K}$ and denote by $[\nu] \in \operatorname{Gal}\left(K^{\mathrm{ab}} / K\right)$ its image under Artin's reciprocity homomorphism and let $f$ and $\omega$ be as above. We denote the action (pull-back) of $v$ on $\varrho_{\omega}$ by ${ }^{v} \varrho_{\omega}$ and obtain

$$
{ }^{v} \varrho_{\omega}(\tilde{f} \circ \Theta)={ }^{\varphi\left(\mathbb{A}_{f}\right)(v)} f\left(x_{\mathrm{cm}}\right) .
$$

But by Proposition 4.4 we know that

$$
\varphi\left(\mathbb{A}_{f}\right)(v)=\bar{\eta}(v) \in \operatorname{GSp}\left(\mathbb{A}_{f}\right),
$$

and due to Shimura's reciprocity law (12) we can conclude that

$$
{ }^{v} \varrho_{\omega}(\tilde{f} \circ \Theta)={ }^{\bar{\eta}(v)} f\left(x_{\mathrm{cm}}\right)=[v]^{-1}\left(f\left(x_{\mathrm{cm}}\right)\right)=[\nu]^{-1}\left(\varrho_{\omega}(\tilde{f} \circ \Theta)\right) \in K^{\mathrm{ab}},
$$

which proves property (vi). For the general case and more details we refer the reader to Section 7.1.

Remark. Our arithmetic subalgebra $\mathscr{A}_{K}$ in the case of $K=\mathbb{Q}(i)$ is essentially the same as in [CMR05]. 
Remark. In a fancy (and very sketchy) way we might say that the two different pictures, one concentrating on the single elliptic curve $A$ and the other on the moduli space of elliptic curves (see the beginning of Section 2.4), are related via the Langlands correspondence. In terms of Langlands's correspondence, the single elliptic curve $A$ lives on the motivic side whereas the moduli space of elliptic curves lives (partly) on the automorphic side. As we used the second picture for our construction of an arithmetic subalgebra, we might say that our construction is automorphic by nature. This explains the fact that we have a "natural" action of the idele class group on our arithmetic subalgebra (see above). Using the recent theory of endomotives (see [CM08], Chapter 4 and in particular p. 551) one can recover the arithmetic subalgebra $A_{K}^{\text {arith }}$ by only using the single elliptic curve $A$. In particular one obtains a natural action of the Galois group. This and more will be elaborated in another paper.

We now concentrate on the general case.

\section{Two Shimura data and a map}

As throughout the paper let $K$ denote a number field and $E$ its maximal $C M$ subfield. We fix an embedding $\tau: K \rightarrow \overline{\mathbb{Q}} \rightarrow \mathbb{C}$ and denote complex conjugation on $\mathbb{C}$ by $\iota$.

To $K$ and $E$, respectively, we will attach a Shimura datum $\delta_{K}$ and $\delta_{\mathrm{Sh}}$, respectively, and show how to construct a morphism $\varphi: \delta_{K} \rightarrow \delta_{\text {Sh }}$ between them. We will freely use the Appendix: every object not defined in the following can be found there or in the references given therein.

Recall that the Serre group attached to $K$ is denoted by $S^{K}$ (cf. C.1), it is a quotient of the algebraic torus $T^{K}$ (defined below), the corresponding quotient map is denoted by $\pi^{K}: T^{K} \rightarrow S^{K}$.

3.1. Protagonist I: $\boldsymbol{S}_{\boldsymbol{K}}$. The 0 -dimensional Shimura datum $\delta_{K}=\left(T^{K}, X_{K}, h_{K}\right)$ (see D.5) is given by the following: the Weil restriction $T^{K}=\operatorname{Res}_{K / \mathbb{Q}}\left(\mathbb{G}_{m, K}\right)$, the discrete and finite set $X_{K}=T^{K}(\mathbb{R}) / T^{K}(\mathbb{R})^{+} \cong \pi_{0}\left(\mathbb{A}_{K, \infty}^{\times}\right)$, and a morphism $h_{K}: \mathbb{S}=\operatorname{Res}_{\mathbb{C} / \mathbb{R}}\left(\mathbb{G}_{m, \mathbb{C}}\right) \rightarrow T_{\mathbb{R}}^{K}$ which is chosen according to the next lemma.

Lemma 3.1. There is a morphism of algebraic groups $h_{K}: \mathbb{S} \rightarrow T_{\mathbb{R}}^{K}$ such that the diagram

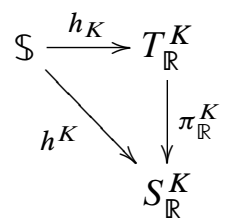

commutes. 
Proof. Remember that $h^{K}: \mathbb{S} \rightarrow S_{\mathbb{R}}^{K}$ is defined as the composition

$$
\mathbb{S} \stackrel{\operatorname{Res}_{\mathbb{C} / \mathbb{R}}\left(\mu^{K}\right)}{\longrightarrow} \operatorname{Res}_{\mathbb{C} / \mathbb{R}}\left(S_{\mathbb{C}}^{K}\right) \stackrel{N m_{\mathbb{C} / \mathbb{R}}}{\longrightarrow} S_{\mathbb{R}}^{K},
$$

where $\mu^{K}: \mathbb{G}_{m, \mathbb{C}} \rightarrow T_{\mathbb{C}}^{K}$ is defined by $\mu^{K}=\pi_{\mathbb{C}}^{K} \circ \mu_{\tau}$ (cf. C.2). Define $h_{K}: \mathbb{S} \rightarrow$ $T_{\mathbb{R}}^{E}$ simply by

$$
\mathbb{S} \stackrel{\operatorname{Res}_{\mathbb{C} / \mathbb{R}}\left(\mu_{\tau}\right)}{\longrightarrow} \operatorname{Res}_{\mathbb{C} / \mathbb{R}}\left(T_{\mathbb{C}}^{K}\right) \stackrel{N m_{\mathbb{C} / \mathbb{R}}}{\longrightarrow} T_{\mathbb{R}}^{K}
$$

For proving our claim it is enough to show that the diagram

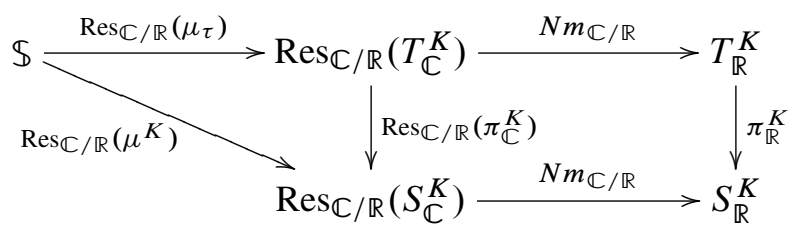

is everywhere commutative.

The triangle on the left is commutative because $\operatorname{Res}_{\mathbb{C} / \mathbb{R}}$ is a functor. Due to Theorem A.1 it is enough to show that rectangle on the right is commutative after applying the functor $X^{*}$ (cf. A.3). Since $\pi^{K}: T^{K} \rightarrow S^{K}$ is defined to be the inclusion $X^{*}\left(S^{K}\right) \subset X^{*}\left(T^{K}\right)$ on the level of characters (cf. C.1), we see that $X^{*}\left(\operatorname{Res}_{\mathbb{C} / \mathbb{R}}\left(\pi_{\mathbb{C}}^{K}\right)\right)$ and $X^{*}\left(\pi_{\mathbb{R}}^{K}\right)$ are inclusions as well and the commutativity follows.

3.2. Protagonist II: $\boldsymbol{S}_{\mathrm{Sh}}$. The construction of the Shimura datum $\boldsymbol{S}_{\mathrm{Sh}}$ in this section goes back to Shimura [Shi00]; we also refer to [Wei]. It is of the form $\delta_{\mathrm{Sh}}=$ $\left(\mathrm{GSp}\left(V_{E}, \psi_{E}\right), \mathbb{U}_{g}^{ \pm}, h_{\mathrm{cm}}\right)$. The symplectic $\mathbb{Q}$-vector space $\left(V_{E}, \psi_{E}\right)$ is defined as follows.

Choose a finite collection of primitive CM types $\left(E_{i}, \Phi_{i}\right), 1 \leq i \leq r$, such that

(i) for all $i$ the reflex field $E_{i}^{*}$ is contained in $E$, i.e., $E_{i}^{*} \subset E$ for all $i$, and

(ii) the natural map (take (34) and apply the universal property from C.1)

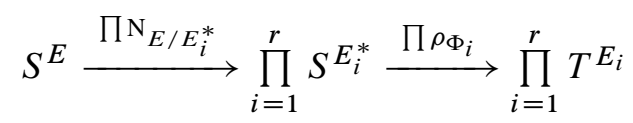

is injective. (Proposition 1.5.1 in [Wei] shows that this is always possible.)

For every $i \in\{1, \ldots, r\}$ we define a symplectic form $\psi_{i}: E_{i} \times E_{i} \rightarrow \mathbb{Q}$ on $E_{i}$ by choosing a totally imaginary generator $\xi_{i}$ of $E_{i}$ (over $\left.\mathbb{Q}\right)$ and setting

$$
\psi_{i}(x, y)=\operatorname{Tr}_{E_{i} / \mathbb{Q}}\left(\xi_{i} x y^{\iota}\right) .
$$

Now we define $\left(V_{E}, \psi_{E}\right)$ as the direct sum of the symplectic spaces $\left(E_{i}, \psi_{i}\right)$. Instead of $\operatorname{GSp}\left(V_{E}, \psi_{E}\right)$ we will sometimes simply write GSp. 
To define the morphism $h_{\mathrm{cm}}$ the essential step is to observe (see [Mi198], Remark 9.2) that the image of the map $\rho_{\Phi_{i}} \circ \mathrm{N}_{E / E_{i}^{*}}: S^{E} \rightarrow T^{E_{i}}$ is contained in the subtorus $\mathcal{T}^{E_{i}}$ of $T^{E_{i}}$, which is defined on the level of $\mathbb{Q}$-points by

$$
\mathcal{T}^{E_{i}}(\mathbb{Q})=\left\{x \in E_{i}^{\times} \mid x x^{\iota} \in \mathbb{Q}^{\times}\right\},
$$

and analogously $T^{E_{i}}(R)$ is defined for an arbitrary $\mathbb{Q}$-algebra $R$. This is an important observation because there is an obvious inclusion of algebraic groups (cf. A.2)

$$
\mathrm{i}: \prod_{i=1}^{r} \mathcal{T}^{E_{i}} \rightarrow \operatorname{GSp}\left(V_{E}, \psi_{E}\right)
$$

whereas there is in general no embedding $\prod T^{E_{i}} \rightarrow \mathrm{GSp}$.

With this in mind we define $h_{\mathrm{cm}}$ as the composition

$$
\mathbb{S} \stackrel{h^{E}}{\longrightarrow} S_{\mathbb{R}}^{E} \stackrel{\prod \mathrm{N}_{E / E_{i}^{*}, \mathbb{R}}}{\longrightarrow} \prod_{i=1}^{r} S_{\mathbb{R}}^{E_{i}^{*}} \stackrel{\prod \rho_{\Phi_{i}, \mathbb{R}}}{\longrightarrow} \prod_{i=1}^{r} \mathcal{T}_{\mathbb{R}}^{E_{i}} \stackrel{\mathfrak{i}_{\mathbb{R}}}{\longrightarrow} \mathrm{GSp}_{\mathbb{R}} .
$$

Write $h_{\mathrm{cm}}^{\prime}: \$ \rightarrow \prod_{i=1}^{r} \mathcal{T}_{\mathbb{R}}^{E_{i}}$ for the composition of the first three arrows.

Remark. (1) By construction, $h_{\mathrm{cm}}$ is a CM point (cf. D.6), which is needed later to construct explicitly abelian extensions $K$. See Section 4.1.

(2) Viewed as a point on the complex analytic space $\mathbb{U}_{g}^{ \pm}$we write $x_{\mathrm{cm}}$ instead of $h_{\mathrm{cm}}$. Further we denote the connected component of $\mathbb{q}_{g}^{ \pm}$containing $x_{\mathrm{cm}}$ by $\mathbb{\sharp} g$, i.e., $x_{\mathrm{cm}} \in \mathbb{H} g$.

Our CM point $h_{\mathrm{cm}}$ enjoys the following properties.

Lemma 3.2. (1) We have $h_{\mathrm{cm}}=\mathrm{i} \circ \prod_{i=1}^{r} h_{\Phi_{i}}($ see (33)).

(2) The field of definition $E\left(x_{\mathrm{cm}}\right)$ of $x_{\mathrm{cm}}$ is equal to the composite of the reflex fields $\widetilde{E}=E_{1}^{*} \ldots E_{r}^{*} \subset E$, i.e., the associated cocharacter $\mu_{\mathrm{cm}}$ of $h_{\mathrm{cm}}$ is defined over $\widetilde{E}$ (see D.6).

(3) The $\mathrm{GSp}(\mathbb{R})$-conjugacy classes of $h_{\mathrm{cm}}$ can be identified with the Siegel upperlower half plane $\mathbb{Q}_{g}^{ \pm}$, for some $g \in \mathbb{N}$ depending on $E$.

Proof. (1) This follows immediately from (36) and (38).

(2) This follows from [Mil04], p. 347, and (1).

(3) For this we refer to the proof of Lemma 3.11 in [Wei].

3.3. The map $\varphi: \boldsymbol{S}_{\boldsymbol{K}} \rightarrow \boldsymbol{S}_{\mathrm{Sh}}$. On the level of algebraic groups $\varphi: T^{K} \rightarrow$ GSp is simply defined as the composition

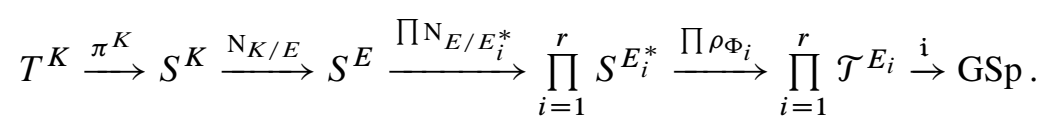


For $\varphi$ being a map between $\delta_{K}$ and $\delta_{\mathrm{Sh}}$ we have to check that the diagram

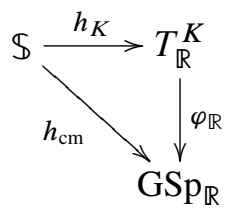

commutes, but this compatibility is built into the construction of $h_{K}$. Using the reflex norm (cf. B.3 and (37)) we can describe $\varphi$ as follows

Lemma 3.3. The map $\varphi: T^{K} \rightarrow \mathrm{GSp}$ is equal to the composition

$$
T^{K} \stackrel{\prod \mathrm{N}_{K / E_{i}^{*}}}{\longrightarrow} \prod_{i=1}^{r} T^{E_{i}^{*}} \stackrel{\prod \mathrm{N}_{\Phi_{i}}}{\longrightarrow} \prod_{i=1}^{r} \mathcal{T}^{E_{i}} \stackrel{i}{\rightarrow} \mathrm{GSp} .
$$

\section{About arithmetic modular functions}

4.1. Introduction. We follow closely [Del79], [MS81] and [Wei]; see also [Hid04]. The reader should be aware of the fact that we are using a normalization of Artin's reciprocity map different from [MS81] and have to correct a "sign error" in [Del79], as pointed out in [Mil04], p. 347.

As usual we denote by $K$ a number field containing a CM subfield and denote by $E$ the maximal CM subfield of $K$. In this section we want to explain how the theory of complex multiplication provides (explicit) abelian extensions of $K$. In general one looks at a CM-point $x \in X$ on a Shimura variety $\operatorname{Sh}(G, X)$ and by the theory of canonical models one knows that the point $[x, 1]$ on the canonical model $M(G, X)$ of $\operatorname{Sh}(G, X)$ is rational over the maximal abelian extension $E(x)^{\text {ab }}$ of the field of definition $E(x)$ of $x$ (see D.6).

In our case we look at Siegel modular varieties $\mathrm{Sh}\left(\mathrm{GSp}, \mathbb{Q}_{g}^{ \pm}\right)$which can be considered as (fine) moduli spaces of Abelian varieties over $\mathbb{C}$ with additional data (level structure, torsion data and polarization). See [Mil04], Chap. 6, for an explanation of this. Each point $x \in \mathbb{Q}_{g}$ corresponds to an Abelian variety $A_{x}$.

In opposite to the case of imaginary quadratic fields, in general the field of definition $E(x)$ is neither contained in $E$ nor in $K$. Therefore, in order to construct abelian extensions of $K$, we have to find an Abelian variety $A_{x}$ such that

$$
E(x) \subset K .
$$

This is exactly the reason for our choice of $x_{\mathrm{cm}} \in \mathbb{U}_{\mathrm{g}}$ because we know (see Lemma $3.2(2)$ ) that

$$
E\left(x_{\mathrm{cm}}\right)=\widetilde{E}=E_{1}^{*} \ldots E_{r}^{*} \subset E \subset K .
$$


Here $x_{\mathrm{cm}}$ corresponds to a product $A_{\mathrm{cm}}=A_{1} \times \cdots \times A_{r}$ of simple Abelian varieties $A_{i}$ with complex multiplication given by $E_{i}$. This construction is the best one can do to generate abelian extensions of $K$ using the theory of complex multiplication. The miracle here is again that the field of definition of $A_{\mathrm{cm}}$ and of its torsion points generate abelian extensions of $E\left(x_{\mathrm{cm}}\right)$. Now to obtain these abelian extensions explicitly one proceeds in complete analogy with the case of $\mathbb{Q}(i)$ explained in Section 2.4, namely rational functions on the connected canonical model $M^{o}$ of the connected Shimura variety $\mathrm{Sh}\left(\mathrm{GSp}, \mathbb{U}_{g}^{ \pm}\right)^{o}$ give rise to arithmetic modular functions on $\mathbb{U}_{g}$ which generate the desired abelian extensions when evaluated at $x_{\mathrm{cm}}$. This will be explained in detail in the following.

\subsection{Working over $\overline{\mathbb{Q}}$}

4.2.1. The field $\mathscr{F}$ of arithmetic automorphic functions. We start with the remark that the reflex field of (GSp, $\mathbb{U}_{g}^{ \pm}$) (cf. D.6) is equal to $\mathbb{Q}$ (see Remark on p. 317).

Remark. This is the second notion of "reflex field". But the reader should not get confused.

Denote by $\Sigma$ the set of arithmetic subgroups $\Gamma$ of $\mathrm{GSp}^{\text {ad }}(\mathbb{Q})^{+}$which contain the image of a congruence subgroup of GSp ${ }^{\text {der }}(\mathbb{Q})$ (see beginning of Appendix D). The connected component of the identity $\mathrm{Sh}^{o}$ of $\mathrm{Sh}=\mathrm{Sh}\left(\mathrm{GSp}, \mathbb{\mathbb { N }} \mathbb{g}_{\mathrm{g}}^{ \pm}\right)$is then given by the inverse limit $\mathrm{Sh}^{o}=\lim _{\Gamma \in \Sigma} \Gamma \backslash \mathbb{M} g$ (cf. D.4).

Denote by $M^{o}=M^{o}\left(\mathrm{GSp}, \mathbb{Q}_{g}^{ \pm}\right)$the canonical model of $S h^{o}$ in the sense of [Del79], 2.7.10, i.e., $M^{o}$ is defined over $\overline{\mathbb{Q}}$. For every $\Gamma \in \Sigma$ the space $\Gamma \backslash \mathbb{Q} g$ is an algebraic variety over $\mathbb{C}$ and $\Gamma \backslash M^{o}$ a model over $\overline{\mathbb{Q}}$.

The field of rational functions $k\left(\Gamma \backslash M^{o}\right)$ on $\Gamma \backslash M^{o}$ is contained in the field of rational functions $k\left(\Gamma \backslash \mathbb{U}_{g}\right)$. Elements in the latter field correspond to meromorphic functions on $\mathbb{U}_{g}$ (now viewed as a complex analytic space) that are invariant under $\Gamma \in \Sigma$.

Following [MS81] we call the field $\mathscr{F}=\bigcup_{\Gamma \in \Sigma} k\left(\Gamma \backslash M^{o}\right)$ the field of arithmetic automorphic functions on $\mathbb{W}_{g}$.

4.2.2. About $\operatorname{Aut}_{\mathbb{Q}}(\mathcal{F})$. The (topological) group $\mathcal{E}$ defined by the extension (see [Del79], 2.5.9)

$$
1 \rightarrow \overline{\mathrm{GSp}^{\mathrm{ad}}(\mathbb{Q})^{+}} \rightarrow \mathcal{E} \stackrel{\sigma}{\rightarrow} \operatorname{Gal}(\overline{\mathbb{Q}} / \mathbb{Q}) \rightarrow 1
$$

is acting continuously on $M^{o}$ ([Del79], 2.7.10) and induces an action on $\mathcal{F}$ by

$$
{ }^{\alpha} f=\sigma(\alpha) \cdot\left(f \circ \alpha^{-1}\right)=(\sigma(\alpha) f) \circ\left(\sigma(\alpha) \alpha^{-1}\right)
$$

(see [MS81], Section 3.2). This is meaningful because $f$ and $\alpha^{-1}$ are both defined over $\overline{\mathbb{Q}}$.

Using this action one can prove the following. 
Theorem 4.1 ([MS81], Theorem 3.3). The map $\mathbb{E} \rightarrow \operatorname{Aut}_{\mathbb{Q}}(\mathcal{F})$ given by (19) identifies $\mathbb{E}$ with an open subgroup of $\operatorname{Aut}_{\mathbb{Q}}(\mathcal{F})$.

4.3. Going down to $\mathbb{Q}^{\text {ab }}$. We said that $M^{o}$ is defined over $\overline{\mathbb{Q}}$ but it is already defined over a subfield $k$ of $\mathbb{Q}^{\mathrm{ab}}$. More precisely $k$ is the fixed field of the kernel of the map $\operatorname{Gal}(\overline{\mathbb{Q}} / \mathbb{Q})^{\mathrm{ab}} \rightarrow \bar{\pi}_{0} \pi(\mathrm{GSp})$ defined in [Del79], 2.6.2.1. Therefore the action of $\mathcal{E}$ on $M^{o}$ factors through the quotient $\overline{\mathcal{E}}$ of $\mathcal{E}$ defined by the following the commutative diagram with exact rows (see [MS81], 4.2 and 4.12, or [Del79], 2.5.3):

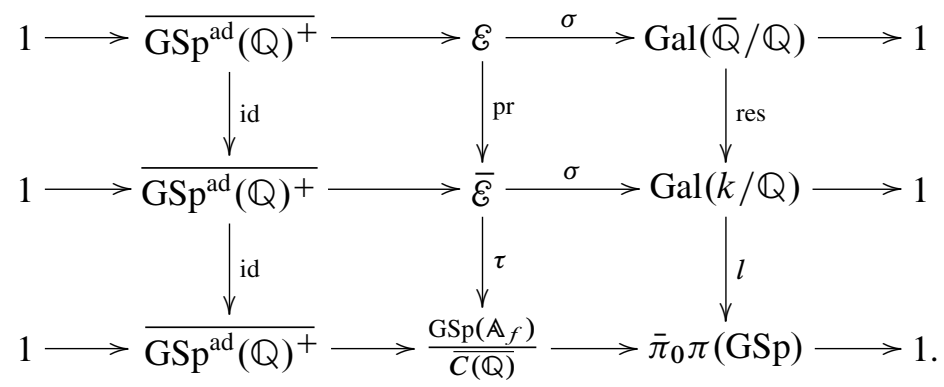

Here $C$ denotes the center of GSp.

Remark. (1) We know that $C(\mathbb{Q})=\mathbb{Q}^{\times}$is discrete in $\operatorname{GSp}\left(\mathbb{A}_{f}\right)$ (cf. [Hid04]).

(2) Because $l$ is injective, $\tau$ is injective as well and we can identify $\overline{\mathscr{E}}$ with an (open) subgroup of $\frac{\mathrm{GSp}\left(\mathbb{A}_{f}\right)}{\mathbb{Q}^{\times}}$.

(3) $\overline{\mathcal{E}}$ is of course depending on $K$, but we suppress this dependence in our notation.

4.3.1. The field $\mathcal{M}$ of arithmetic modular functions. Let $f \in \mathscr{F}$ be a rational function, i.e., $f$ is a rational function on $\Gamma \backslash M^{o}$, for some $\Gamma \in \Sigma$. We call $f$ an arithmetic modular function if it is rational over $k$, and meromorphic at the cusps (when viewed on the corresponding complex analytic space). Compare this to [Wei], Section 3.4, or [Mil98], p. $35 \mathrm{ff}$.

Definition 4.1. The subfield of $\mathscr{F}$ generated by all arithmetic modular functions is denoted by $\mathcal{M}$. Further we denote by $\mathcal{M}^{\mathrm{cm}}$ the subring of $\mathcal{M}$ of all arithmetic modular functions which are defined in $x_{\mathrm{cm}}$.

The importance of $\mathcal{M}^{\mathrm{cm}}$ for our purposes is explained (see [Mil98], Lemma 14.4, and [Wei], Lemma 3.11) by

Theorem 4.2. Let $A_{\mathrm{cm}}$ denote the abelian variety corresponding to $x_{\mathrm{cm}}(c f$. Theorem 4.1).

Denote by $K^{A_{\mathrm{cm}}}$ the field extension of $K$ obtained by adjoining the field of definition of $A_{\mathrm{cm}}$ and all of its torsion points. 
Further denote by $K^{\mathcal{M}}$ the field extension of $K$ obtained by adjoining the values $f\left(x_{\mathrm{cm}}\right)$, for $f \in \mathcal{M}^{\mathrm{cm}}$.

Finally denote by $K^{c}$ the composition of $K$ with the fixed field of the image of the Verlagerungsmap $\operatorname{Ver}: \operatorname{Gal}\left(F^{\mathrm{ab}} / F\right) \rightarrow \operatorname{Gal}\left(E^{\mathrm{ab}} / E\right)$, where $F$ is the maximal totally real subfield of $E$. Then we have the equality

$$
K^{c}=K^{A_{\mathrm{cm}}}=K^{\mathcal{M}} .
$$

Remark. Notice we are not simply using the field of arithmetic automorphic functions as considered by Shimura - see [MS81], Section 4.8, for his definition - because the exact size of the abelian extension obtained by using these functions is not clear (at least to the author). It is clear that the field of Shimura is contained in $K^{\mathcal{M}}$ and we guess that it should generate the same extension $K^{c}$ of $K$.

4.3.2. About $\operatorname{Aut}_{\mathbb{Q}}(\mathcal{M})$. It is clear that $\mathcal{M}$ is closed under the action of $\overline{\mathcal{E}}$ (see [MS81], Section 3.2 or Section 4.4) and therefore we obtain a continuous map

$$
\overline{\mathcal{E}} \rightarrow \operatorname{Aut}_{\mathbb{Q}}(\mathcal{M})
$$

given like above by

$$
{ }^{\alpha} f=\sigma(\alpha) \cdot\left(f \circ \alpha^{-1}\right)=(\sigma(\alpha) f) \circ\left(\sigma(\alpha) \alpha^{-1}\right) .
$$

In particular $\mathrm{GSp}^{\mathrm{ad}}(\mathbb{Q})^{+}$is acting on $\mathcal{M}$ by

$$
{ }^{\alpha} f=f \circ \alpha^{-1} \text {. }
$$

\subsection{The reciprocity law at $x_{\mathrm{cm}}$. Write}

$$
\mu_{\mathrm{cm}}: \mathbb{G}_{m, \mathbb{C}} \rightarrow S_{\mathbb{C}}^{E} \stackrel{\left(h_{\mathrm{cm}}^{\prime}\right) \mathbb{C}}{\longrightarrow} \prod_{i=1}^{r} \mathcal{T}^{E_{i, \mathbb{C}}} \stackrel{\mathrm{i}}{\rightarrow} \mathrm{GSp}_{\mathbb{C}}
$$

for the associated cocharacter of $h_{\mathrm{cm}}$ (cf. D.6). From Lemma 3.2(1) we know that $h_{\mathrm{cm}}=\mathrm{i} \circ \prod h_{\phi_{i}}$ and therefore $\mu_{\mathrm{cm}}=\mathfrak{i} \circ \prod \mu_{\phi_{i}}$. Because $\mu_{\phi_{i}}$ is defined over $E_{i}^{*}$ the cocharacter $\mu_{\mathrm{cm}}^{\prime}=\prod \mu_{\phi_{i}}$ is defined over $\widetilde{E}=E_{1}^{*} \ldots E_{r}^{*} \subset E \subset K$. To simplify the notation set $\mathcal{T}=\prod_{i=1}^{r} \mathcal{T}^{E_{i}}$. Define the morphism

$$
\eta: T^{K} \rightarrow \mathrm{GSp}
$$

as composition of

$$
T^{K} \stackrel{\operatorname{Res}_{K / \mathbb{Q}}\left(\mu_{\mathrm{cm}}^{\prime}\right)}{\longrightarrow} \operatorname{Res}_{K / \mathbb{Q}}\left(\mathcal{T}_{K}\right) \stackrel{N m_{K / \mathbb{Q}}}{\longrightarrow} \mathcal{T} \stackrel{\mathfrak{i}}{\rightarrow} \mathrm{GSp} .
$$

If we identify $\overline{\mathcal{E}}$ with an (open) subset of $\frac{\mathrm{GSp}\left(\mathbb{A}_{f}\right)}{\mathbb{Q}^{\times}}$using $\tau$ (see (20)), one can show (see [MS81], Section 4.5, or [Del79], 2.6.3) that $\eta(\mathbb{A}): \mathbb{A}_{K}^{\times} \rightarrow \mathrm{GSp}(\mathbb{A})$ induces, by $v \mapsto \eta\left(\mathbb{A}_{f}\right)(v) \bmod \mathbb{Q}^{\times}$, a group homomorphism

$$
\bar{\eta}: \mathbb{A}_{K}^{\times} \rightarrow \overline{\mathcal{E}} .
$$


If we denote by $[v] \in \operatorname{Gal}\left(K^{\mathrm{ab}} / K\right)$ the image of $v \in \mathbb{A}_{K}^{\times}$under Artin's reciprocity map one can show (cf. [MS81], Section 4.5) that (cf. (20))

$$
\sigma(\bar{\eta}(v))=\left.[v]^{-1}\right|_{k} .
$$

Remark. The careful reader will ask why it is allowed to define $\eta$ using the extension $K$ of the field of definition of the cocharacter $\mu_{\mathrm{cm}}$ given by $\widetilde{E}$ because our reference [MS81] uses $\widetilde{E}$ to define $\eta$. The explanation for this is given by Lemma 4.5 and standard class field theory.

Now we are able to state the reciprocity law

Theorem 4.3 ([MS81], Theorem 4.6 and Section 4.10). Let $v \in \mathbb{A}_{K}^{\times}$and $f \in \mathcal{M}^{\mathrm{cm}}$. Then $f\left(x_{\mathrm{cm}}\right)$ is rational over $K^{\mathrm{ab}}$. Further ${ }^{\bar{\eta}(v)} f$ is defined in $x_{\mathrm{cm}}$ and

$$
{ }^{\bar{\eta}(v)} f\left(x_{\mathrm{cm}}\right)=[v]^{-1}\left(f\left(x_{\mathrm{cm}}\right)\right) .
$$

Proof. We simply reproduce the argument given in the proof of [MS81], Theorem 4.6. The first assertion is clear by the definition of the canonical model (cf. D.6) and the other two assertions follow from the following calculation.

Regard the special point $x_{\mathrm{cm}}$ as a point on the canonical model $\left[x_{\mathrm{cm}}, 1\right] \in M^{o}$. The action of $\bar{\eta}(v)^{-1}$ is given by $\bar{\eta}(v)^{-1}\left[x_{\mathrm{cm}}, 1\right]=\sigma\left(\bar{\eta}(v)^{-1}\right)\left[x_{\mathrm{cm}}, \eta(v)\right]$ and further we know $\left[x_{\mathrm{cm}}, \eta(v)\right]=[v]^{-1}\left[x_{\mathrm{cm}}, 1\right]$ (by (40)). Therefore we obtain

$$
\begin{aligned}
& { }^{\bar{\eta}(v)} f\left(x_{\mathrm{cm}}\right)=\sigma(\bar{\eta}(v)) \cdot\left(f \circ \bar{\eta}(v)^{-1}\right)\left(\left[x_{\mathrm{cm}}, 1\right]\right) \\
& =\left(\sigma(\bar{\eta}(v) f) \circ\left(\sigma(\bar{\eta}(v)) \bar{\eta}(v)^{-1}\right)\left(\left[x_{\mathrm{cm}}, 1\right]\right)\right. \\
& =\left(\sigma(\bar{\eta}(v) f) \circ\left(\sigma(\bar{\eta}(v)) \sigma(\bar{\eta}(v))^{-1}\right)\left(\left[x_{\mathrm{cm}}, \eta(v)\right]\right)\right. \\
& \stackrel{(23)}{=}\left(\left.[v]^{-1}\right|_{k} f\right)\left([v]^{-1}\left[x_{\mathrm{cm}}, 1\right]\right)=[v]^{-1}\left(f\left(\left[x_{\mathrm{cm}}, 1\right]\right)\right) \\
& =[v]^{-1}\left(f\left(x_{\mathrm{cm}}\right)\right) \text {. }
\end{aligned}
$$

The next observation is one of the key ingredients in our construction of the arithmetic subalgebra.

Proposition 4.4. The two maps of algebraic groups $\varphi$ and $\eta$ are equal.

Proof. This is an immediate corollary of Proposition C.1, Lemma 3.3, the compatibility properties of the norm map and the next simple lemma.

Lemma 4.5. Let $(L, \phi)$ a CM type and $L^{\prime}$ a finite extension of the reflex field $L^{*}$. Then the following diagram is commutative:

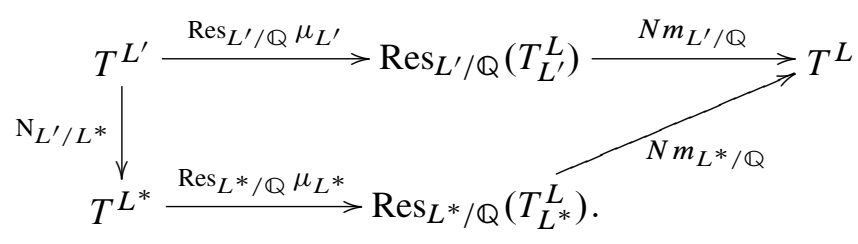


Now having at hand all the number-theoretic ingredients that are needed, we can move on to the "operator-theoretic" part of this paper.

\section{On Bost-Connes-Marcolli systems}

We review very briefly the general construction of $C^{*}$-dynamical systems, named Bost-Connes-Marcolli systems, as given in [HP05].

5.1. BCM pairs. A BCM pair $(\mathscr{D}, \mathscr{L})$ is a pair consisting of a $B C M$ datum $\mathscr{D}=$ $(G, X, V, M)$ together with a level structure $\mathscr{L}=\left(L, \Gamma, \Gamma_{M}\right)$ of $\mathscr{D}$.

A BCM datum is a Shimura datum $(G, X)$ together with an enveloping algebraic semigroup $M$ and a faithful representation $\phi: G \rightarrow \operatorname{GL}(V)$ such that $\phi(G) \subset M \subset$ $\operatorname{End}(V)$. Here $V$ denotes a $\mathbb{Q}$-vector space of finite dimension.

A level structure $\mathscr{L}$ of $\mathscr{D}$ consists of a lattice $L \subset V$, a compact open subgroup $\Gamma \subset G\left(\mathbb{A}_{f}\right)$ and a compact open semigroup $\Gamma_{M} \subset M\left(\mathbb{A}_{f}\right)$ such that $\phi(\Gamma) \subset \Gamma_{M}$ and $\Gamma_{M}$ stabilizes $L \otimes_{\mathbb{Z}} \widehat{\mathbb{Z}}$.

Remark. In [HP05], Definition 3.1, a more general notion of Shimura datum is allowed than ours given in Appendix D.

To every BCM datum $\mathcal{D}$ and lattice $L \subset V$ one can associate the following socalled maximal level structure to obtain a BCM pair by setting $\Gamma_{M}=M\left(\mathbb{A}_{f}\right) \cap$ $\operatorname{End}\left(L \otimes_{\mathbb{Z}} \widehat{\mathbb{Z}}\right)$ and $\Gamma=\phi^{-1}\left(\Gamma_{M}^{\times}\right)$.

The level structure $\mathscr{L}$ is called fine if $\Gamma$ is acting freely on $G(\mathbb{Q}) \backslash\left(X \times G\left(\mathbb{A}_{f}\right)\right)$.

Remark. For the definition of the topology of $G\left(\mathbb{A}_{f}\right)$ and $M\left(\mathbb{A}_{f}\right)$ we refer the reader to [PR94]. Especially one can show that $\phi\left(\mathbb{A}_{f}\right): G\left(\mathbb{A}_{f}\right) \rightarrow M\left(\mathbb{A}_{f}\right)$ is a continuous map (cf. [PR94], Lemma 5.2).

\subsection{Quotient maps attached to BCM pairs. Let $(\mathscr{D}, \mathscr{L})$ a $\mathrm{BCM}$ pair.}

5.2.1. The BCM groupoid. There is a partially defined action of $G\left(\mathbb{A}_{f}\right)$ on the direct product $\Gamma_{M} \times \operatorname{Sh}(G, X)$ given by

$$
g(\rho,[z, l])=\left(g \rho,\left[z, l g^{-1}\right]\right),
$$

where we suppressed the morphism $\phi$. Using this the BCM groupoid $U$ is the topological groupoid (using the notation given in (4)) defined by

$$
U=G\left(\mathbb{A}_{f}\right) \boxplus\left(\Gamma_{M} \times \operatorname{Sh}(G, X)\right) .
$$

There is an action of the group $\Gamma \times \Gamma$ on $U$ given by

$$
\left(\gamma_{1}, \gamma_{2}\right)(g, \rho,[z, l])\left(\gamma_{1} g \gamma_{2}^{-1}, \gamma_{2} \rho,\left[z, l \gamma_{2}^{-1}\right]\right) .
$$


We denote the quotient by $Z=(\Gamma \times \Gamma) \backslash U$ and obtain a natural quotient map

$$
U \rightarrow Z \text {. }
$$

We denote elements in $Z$ by $[g, \rho,[z, l]]$.

Remark. In general the quotient $Z$ is not a groupoid anymore; see [HP05], Definition 4.2.1.

5.2.2. The positive BCM groupoid. Assume that the Shimura datum $(G, X)$ of our BCM pair satisfies (SV5) (cf. D.1). Moreover we choose a connected component $X^{+}$of $X$ and set $G(\mathbb{Q})^{+}=G(\mathbb{Q}) \cap G(\mathbb{R})^{+}$, where $G(\mathbb{R})^{+}$denotes the connected component of the identity of $G(\mathbb{R})$. Then $G(\mathbb{Q})^{+}$is acting naturally on $X^{+}$, because $X^{+}$can be regarded as a $G(\mathbb{R})^{+}$-conjugacy class (see D.4). Now we can consider the positive (BCM) groupoid $U^{+}$which is the topological groupoid given by

$$
U^{+}=G(\mathbb{Q})^{+} \boxplus\left(\Gamma_{M} \times X^{+}\right) .
$$

If we set $\Gamma^{+}=\Gamma \cap G(\mathbb{Q})^{+}$we see further that $\Gamma^{+} \times \Gamma^{+}$is acting on $U^{+}$by

$$
\left(\gamma_{1}, \gamma_{2}\right)(g, \rho, z)=\left(\gamma_{1} g \gamma_{2}^{-1}, \gamma_{2} \rho, \gamma_{2} z\right) .
$$

We denote the quotient by $Z^{+}=\left(\Gamma^{+} \times \Gamma^{+}\right) \backslash U^{+}$and obtain another quotient map

$$
U^{+} \rightarrow Z^{+} \text {. }
$$

There is a natural equivariant morphism of topological groupoids

$$
U^{+} \rightarrow U
$$

given by $(g, \rho, z) \mapsto(g, \rho,[z, 1])$, inducing a commutative diagram

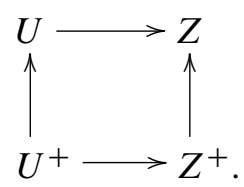

The following criterion given in [HP05], Section 5.1, will be crucial for our approach

Criterion 5.1. If the natural map $G(\mathbb{Q}) \cap \Gamma \rightarrow G(\mathbb{Q}) / G(\mathbb{Q})^{+}$is surjective and $\left|G(\mathbb{Q}) \backslash G\left(\mathbb{A}_{f}\right) / \Gamma\right|=1$, then the natural morphism (27) induces a homeomorphism of topological spaces

$$
Z^{+} \rightarrow Z \text {. }
$$


Remark. (1) In other words the two conditions of the criterion simply mean that we have a decomposition of the form $G\left(\mathbb{A}_{f}\right)=G(\mathbb{Q})^{+} \cdot \Gamma$.

(2) The inverse $Z \rightarrow Z^{+}$of the above homeomorphism is given explicitly as follows. By using the first remark we can write every $l \in G\left(\mathbb{A}_{f}\right)$ as a product $l=\alpha \beta$ with $\alpha \in G(\mathbb{Q})^{+}$and $\beta \in \Gamma$ (this decomposition is unique up to an element in $\left.\Gamma^{+}=\Gamma \cap G(\mathbb{Q})^{+}\right)$. In particular every element $[g, \rho,[z, l]] \in Z$ can be written as $[g, \rho,[z, l]]=\left[g \beta^{-1}, \beta \rho,\left[\alpha^{-1} z, 1\right]\right]$ and under the inverse of the above homeomorphism this element is sent to $\left[g \beta^{-1}, \beta \rho, \alpha^{-1} z\right] \in Z^{+}$.

5.2.3. The adjoint BCM algebra. Let us denote by $C$ the center of $G$ and assume further that $\phi(\overline{C(\mathbb{Q})})$ is a normal subsemigroup of $M\left(\mathbb{A}_{f}\right)$. The adjoint group $G^{\text {ad }}$ of $G$ is the quotient of $G$ by its center $C$ (in the sense of algebraic groups, see [Wat79]). Let us define the semigroup $\Gamma_{M}^{\text {ad }}$ to be the quotient of $\Gamma_{M}$ by the normal subsemigroup $\phi(\overline{C(\mathbb{Q})}) \cap \Gamma_{M}$ and remember that $X^{+}$can be naturally regarded as a $G^{\text {ad }}(\mathbb{R})^{+}$-conjugacy class (see D.4). With this in hand we define the adjoint (BCM) groupoid $U^{\text {ad }}$ to be the topological groupoid

$$
U^{\mathrm{ad}}=G^{\mathrm{ad}}(\mathbb{Q})^{+} \boxplus\left(\Gamma_{M}^{\mathrm{ad}} \times X^{+}\right) .
$$

It is known that the projection $G \longrightarrow G^{\text {ad }}$ induces a surjective group homomorphism $\pi^{\text {ad }}: G(\mathbb{Q})^{+} \rightarrow G^{\text {ad }}(\mathbb{Q})^{+}$(see [Mil04], Proposition 5.1). Setting $\Gamma^{\text {ad }}=\pi^{\text {ad }}\left(\Gamma^{+}\right)$ we see immediately that $\Gamma^{\text {ad }} \times \Gamma^{\text {ad }}$ is acting on $U^{\text {ad }}$ exactly as in (26). We obtain yet another quotient map

$$
U^{\text {ad }} \rightarrow Z^{\text {ad }}
$$

Using the two projections $\Gamma_{M} \rightarrow \Gamma_{M}^{\text {ad }}$ and $\pi^{\text {ad }}$ there is by construction an obvious equivariant morphism of topological groupoids

$$
U^{+} \rightarrow U^{\text {ad }}
$$

which induces (together with (28)) a commutative diagram

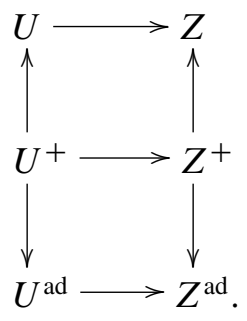

5.3. BCM algebras and systems. Let $(\mathscr{D}, \mathscr{L})$ be a $B C M$ pair. The $B C M$ algebra $H=H_{\mathscr{D}, \mathscr{L}}$ is defined to be the set of compactly supported, continuous function on the quotient $Z=(\Gamma \times \Gamma) \backslash U$ of the BCM groupoid $U$, i.e.,

$$
H=C_{c}(Z) \text {. }
$$


By viewing functions in $H$ as $\Gamma \times \Gamma$-invariant functions on the groupoid $U$, we can equip $H$ with the structure of a $*$-algebra by using the usual convolution and involution on $U$ (like in the construction of groupoid $C^{*}$-algebras). We refer to [HP05], Section 4.3.2, for the details. After completing $H$ in a suitable norm we obtain a $C^{*}$-algebra $A$ (see [HP05], Section 6.2). Further there is a time evolution $\left(\sigma_{t}\right)_{t \in \mathbb{R}}$ on $H$ resp. $A$ so that we end up with the BCM system $\mathcal{A}=\mathcal{A}_{\mathcal{D}, \mathscr{L}}$ given by the $C^{*}$-dynamical system

$$
\mathcal{A}=\left(A,\left(\sigma_{t}\right)_{t \in \mathbb{R}}\right)
$$

associated with the BCM pair $(\mathscr{L}, \mathscr{D})$. For the general definition of the time evolution we refer to [HP05], Section 4.4. We will state the time evolution $\left(\sigma_{t}\right)_{t \in \mathbb{R}}$ only in the case of our BC-type systems $\mathcal{A}_{K}$ (cf. Section 6.1.3).

Remark. In complete analogy one might construct a positive respectively adjoint BCM system, but we do not need this.

5.4. On symmetries of BCM algebras. In Section 4.5 of [HP05] the authors define symmetries of BCM algebras, but for our purpose we need to deviate from their definition in order to be in accordance with the definition of symmetries for BC-type systems given in [LLN09].

Let $(\mathscr{D}, \mathscr{L})$ be a BCM pair with fine level structure (see Section 5.1) and recall (D.2) that there is a natural right action of $G\left(\mathbb{A}_{f}\right)$ on the Shimura variety $\operatorname{Sh}(G, X)$ which is denoted by $m[z, l]=[z, l m]$. Define the subgroup $G_{\Gamma}\left(\mathbb{A}_{f}\right)=\{g \in$ $G\left(\mathbb{A}_{f}\right) \mid g \gamma=\gamma g$ for all $\left.\gamma \in \Gamma\right\}$. Further, if we denote by $C$ the center of $G$, the group $C(\mathbb{R})$ is acting on $\operatorname{Sh}(G, X)$ by $c[z, l]=[c z, l]$. We end up with a right action of $G_{\Gamma}\left(\mathbb{A}_{f}\right) \times C(\mathbb{R})$ as symmetries on the BCM algebra $H_{\mathscr{D}, \mathscr{L}}$ given on a function $f \in C_{c}(Z)$ by

$$
{ }^{(m, c)} f(g, \rho,[z, l])=f(g, \rho,[c z, l m]) .
$$

Remark. If $G\left(\mathbb{A}_{f}\right)$ is a commutative group and we have a decomposition $G\left(\mathbb{A}_{f}\right)=$ $G(\mathbb{Q}) \cdot \Gamma$ then it is immediate that our symmetries agree with the ones defined in [HP05], Section 4.5.

\section{Two BCM pairs and a map}

In this section we will apply the constructions from the last section to our Shimura data $\delta_{K}=\left(T^{K}, X_{K}, h_{K}\right)$ and $\oint_{\mathrm{Sh}}=\left(\mathrm{GSp}\left(V_{E}, \psi_{E}\right)\right.$, 田,$\left.h_{\mathrm{cm}}\right)$ from Section 3 and show how the two resulting systems can be related.

6.1. Costume I: $\left(\mathscr{D}_{K}, \mathscr{L}_{K}\right)$ and $\mathcal{A}_{K}$. In this section $K$ is an arbitrary number field. 
6.1.1. The BCM groupoid $\left(\mathscr{D}_{K}, \mathscr{L}_{K}\right)$. Let us recall the BCM pair $\left(\mathscr{D}_{K}, \mathscr{L}_{K}\right)$ from [HP05], Definition 5.5, attached to $\delta_{K}$. It is given by

$$
\left(D_{K}, \mathscr{L}_{K}\right)=\left(\left(\mathcal{S}_{K}, K, M^{K}\right),\left(\mathcal{O}_{K}, \hat{\mathcal{O}}_{K}^{\times}, \widehat{\mathcal{O}}_{K}\right)\right),
$$

where the algebraic semigroup $M^{K}$ is represented by the functor which assigns to a $\mathbb{Q}$-algebra $R$ the semigroup of $\mathbb{Q}$-algebra homomorphisms $\operatorname{Hom}(K[X], K \otimes \mathbb{Q} R)$. By definition we have that $M^{K}(R)^{\times}=T^{K}(R)$ for every $\mathbb{Q}$-algebra $R$, which gives an embedding $\phi: T^{K} \rightarrow M^{K}$. (It will be convenient to set $\Gamma_{K}=\hat{\mathcal{O}}_{K}^{\times}$.)

6.1.2. The quotient map $U_{K} \rightarrow Z_{K}$. The corresponding BCM groupoid, denoted by $U_{K}$, is given by

$$
U_{K}=T^{K}\left(\mathbb{A}_{f}\right) \boxplus\left(\hat{\mathcal{O}}_{K} \times \operatorname{Sh}\left(S_{K}\right)\right)
$$

with $\Gamma_{K}^{2}=\hat{\mathcal{O}}_{K}^{\times} \times \hat{\mathcal{O}}_{K}^{\times}$acting as in (25). We denote the quotient of this action by

$$
Z_{K}=\Gamma_{K}^{2} \backslash U_{K}
$$

6.1.3. The time evolution. Following [HP05], Section 7.3, the time evolution $\left(\sigma_{t}\right)_{t \in \mathbb{R}}$ on the BCM algebra $H_{K}=C_{c}\left(Z_{K}\right)$ is given as follows. Denote by $\mathfrak{N}=N_{K / \mathbb{Q}}: \mathbb{A}_{K, f}^{\times} \rightarrow \mathbb{R}$ the usual idele norm. Let $f \in H_{K}$ be a function, then we have

$$
\sigma_{t}(f)(g, \rho,[z, l])=\mathfrak{N}(g)^{i t} f(g, \rho,[z, l]) .
$$

6.1.4. On symmetries. First, from [Del79], 2.2.3, we know that there is an isomorphism between $\mathrm{Sh}=\mathrm{Sh}\left(T^{K}, X_{K}, h_{K}\right)$ and $\pi_{0}\left(C_{K}\right)$. By class field theory the latter space $\pi_{0}\left(C_{K}\right)=C_{K} / D_{K}$ is identified with the Galois group $\operatorname{Gal}\left(K^{\mathrm{ab}} / K\right)$ of the maximal abelian extension of $K$ using the Artin reciprocity homomorphism. Under this identification the natural action of $T^{K}\left(\mathbb{A}_{f}\right)=\mathbb{A}_{K, f}^{\times}$on Sh corresponds simply to the Artin reciprocity map, i.e., if $v$ is a finite idele in $T\left(\mathbb{A}_{f}\right)$ and $\omega_{1}=[g, l] \in \mathrm{Sh}$ corresponds to the identity in $\operatorname{Gal}\left(K^{\mathrm{ab}} / K\right)$, then $v \omega_{1}=[g, l \nu]$ corresponds to the image $[v]$ in $\operatorname{Gal}\left(K^{\mathrm{ab}} / K\right)$ of $v$ under Artin's reciprocity map.

Now, because $T^{K}$ is commutative, we see that $C\left(\mathbb{A}_{f}\right) \times C(\mathbb{R})=T^{K}(\mathbb{A})=\mathbb{A}_{K}^{\times}$ is acting by symmetries on $H_{K}$. By what we just said this action is simply given by the natural map $\mathbb{A}_{K}^{\times} \rightarrow \pi_{0}\left(C_{K}\right)=C_{K} / D_{K}$ so that we obtain (tautologically) the desired action of $C_{K} / D_{K} \cong \mathrm{Gal}\left(K^{\mathrm{ab}} / K\right)$ on $H_{K}$.

Remark. The reader should notice that in the case of an imaginary quadratic number field $K$ our symmetries do not agree with the symmetries defined [CMR05] (except when the class number of $K$ is equal to one, where the two definitions agree). For a short discussion on this matter we refer the reader to the Remark on p. 308. 
6.1.5. About extremal $\mathrm{KMS}_{\infty}$-states of $\mathcal{A}_{\boldsymbol{K}}$. We refer to [CM08], p. $445 \mathrm{ff}$., or [BR81] for the notion of extremal $\mathrm{KMS}_{\infty}$-states.

Let $\mathcal{A}_{K}=\left(A_{K},\left(\sigma_{t}\right)_{t \in \mathbb{R}}\right)$ denote the corresponding BCM system (cf. Section 5.3). In [LLN09], Theorem 2.1 (vi), it is shown that the set $\varepsilon_{\infty}$ of extremal $\mathrm{KMS}_{\infty}$-states of $\mathcal{A}_{K}$ is indexed by the set $\mathrm{Sh}=\mathrm{Sh}\left(T^{K}, X_{K}, h_{K}\right)$ and the extremal $\mathrm{KMS}_{\infty}$-state $\varrho_{\omega}$ associated with $\omega \in$ Sh is given on a function $f \in H_{K}$ by evaluation, namely

$$
\varrho_{\omega}(f)=f(1,1, \omega) .
$$

Remark. (1) It follows immediately that the symmetry group $C_{K} / D_{K}$ is acting free and transitively on the set of extremal $\mathrm{KMS}_{\infty}$-states.

(2) Using the definition of symmetries given in [HP05] we obtain another action of $C_{K} / D_{K}$ on $H_{K}$ but in general this will not induce a free and transitive action on the extremal $\mathrm{KMS}_{\infty}$-states. In fact the two different actions of $C_{K} / D_{K}$ on $H_{K}$ given in [LLN09] resp. [HP05] are equivalent if and only if the class number $h_{K}$ of $K$ is equal to 1 . This follows directly from the fact that if (and only if) $h_{K}=1$ then already $\hat{\mathcal{O}}_{K}^{\times}$surjects onto $\operatorname{Gal}\left(K^{\mathrm{ab}} / K\right)$.

All put together we have the following result.

Theorem 6.1 ([HP05] and [LLN09]). Let $K$ be an arbitrary number field. Then the $B C M$ system $\mathcal{A}_{K}=\left(A_{K},\left(\sigma_{t}\right)_{t \in \mathbb{R}}\right)$ is a BC-type system (cf. p. 276).

\subsection{Costume II: $\left(\mathscr{D}_{\mathrm{Sh}}, \mathscr{L}_{\mathrm{Sh}}\right)$}

6.2.1. The BCM groupoid $\left(\mathscr{D}_{\mathrm{Sh}}, \mathscr{L}_{\mathrm{Sh}}\right)$. Recall the construction of the symplectic vector space $\left(V_{E}, \psi_{E}\right)$ (see Section 3.2). We still have some freedom in specifying the totally imaginary generators $\xi_{i}$ of the (primitive) CM fields $E_{i}$, which in turn define the symplectic form $\psi_{E}$ (cf. (14)). Let us denote by $L_{E}$ the lattice $L_{E}=$ $\bigoplus_{i=1}^{r} \mathcal{O}_{E_{i}} \subset V_{E}=\bigoplus_{i=1}^{r} E_{i}$. We now fix generators $\xi_{i}$ according to the following lemma.

Lemma 6.2. For each $i \in\{1, \ldots, r\}$ there exists a totally imaginary generator $\xi_{i} \in E_{i}$, such that the associated symplectic vector space $\left(V_{E}, \psi_{E}\right)$ is integral with respect to $L_{E}$, i.e., there exists a symplectic basis $\left\{e_{j}\right\}$ for $\left(V_{E}, \psi_{E}\right)$ such that $e_{j} \in L_{E}$ for each $j$.

Proof. For each $i$, choose any totally imaginary generator $\tilde{\xi}_{i} \in E_{i}$ and regard the associated symplectic form $\widetilde{\psi}_{E}$ on $V_{E}$ (see (14)). It is known that there exists a symplectic basis $\left\{\tilde{e}_{j}\right\}$ for $\left(V_{E}, \tilde{\psi}_{E}\right)$. Now for each $j$ there exists a $q_{j} \in \mathbb{N}$ such that $e_{j}=q_{j} \tilde{e}_{j} \in L_{E}$ because $E_{i}=\mathcal{O}_{E_{i}} \otimes_{\mathbb{Z}} \mathbb{Q}$. Set $q=\prod_{j} q_{j}$ and define the symplectic form $\psi_{E}$ on $V_{E}$ by using, for every $i$, the totally imaginary generators $\xi_{i}=q^{-2} \tilde{\xi}_{i} \in E_{i}$. By construction it is now clear that $\left\{e_{j}\right\}$ is an integral symplectic basis for $\left(V_{E}, \psi_{E}\right)$. 
Now having fixed our Shimura datum $\S_{\mathrm{Sh}}=\left(\operatorname{GSp}\left(V_{E}, \psi_{E}\right)\right.$, 㱐 $\left.{ }_{g}, h_{\mathrm{cm}}\right)$ we define the BCM pair $\left(\mathscr{D}_{\mathrm{Sh}}, \mathscr{L}_{\mathrm{Sh}}\right)$ equipped with the maximal level structure (cf. Section 5.1) with respect to the lattice $L_{E}$ by

$$
\left(\mathscr{D}_{\mathrm{Sh}}, \mathscr{L}_{\mathrm{Sh}}\right)=\left(\left(\oint_{\mathrm{Sh}}, V_{E}, \mathrm{MSp}\right),\left(L_{E}, \Gamma_{\mathrm{Sh}}, \Gamma_{\mathrm{Sh}, M}\right)\right),
$$

where the algebraic semigroup $\operatorname{MSp}=\operatorname{MSp}\left(V_{E}, \psi_{E}\right)$ is represented by the functor which assigns to a $\mathbb{Q}$-algebra $R$ the semigroup

$$
\begin{array}{r}
\operatorname{MSp}(R)=\left\{f \in \operatorname{End}_{R}\left(V_{E} \otimes_{\mathbb{Q}} R\right) \mid \text { there exists } v(f) \in R\right. \text { such that } \\
\left.\psi_{E, R}(f(x), f(y))=v(f) \psi_{E, R}(x, y) \text { for all } x, y\right\} .
\end{array}
$$

It is clear by definition (compare A.2) that $\operatorname{MSp}(R)^{\times}=\mathrm{GSp}(R)$, which defines a natural injection $\phi: \mathrm{GSp} \rightarrow \mathrm{MSp}$.

6.2.2. Some quotient maps. We denote the corresponding BCM groupoid by

$$
U_{\mathrm{Sh}}=\operatorname{GSp}\left(\mathbb{A}_{f}\right) \boxplus\left(\Gamma_{\mathrm{Sh}, M} \times \operatorname{Sh}\left(\delta_{\mathrm{Sh}}\right)\right),
$$

where the group $\Gamma_{\mathrm{Sh}}^{2}=\Gamma_{\mathrm{Sh}} \times \Gamma_{\mathrm{Sh}}$ is acting as usual. We denote the quotient of $U_{\mathrm{Sh}}$ by this action by

$$
Z_{\mathrm{Sh}}=\Gamma_{\mathrm{Sh}}^{2} \backslash U_{\mathrm{Sh}} .
$$

Due to D.3 and part (1) of the Remark on p. 295 we are allowed to consider the positive and adjoint BCM groupoid, which we denote by $U_{\mathrm{Sh}}^{+}$and $U_{\mathrm{Sh}}^{\mathrm{ad}}$, respectively. The corresponding quotients are denoted analogously by $Z_{\mathrm{Sh}}^{+}=\left(\Gamma_{\mathrm{Sh}}^{+}\right)^{2} \backslash U_{\mathrm{Sh}}^{+}$and $Z_{\mathrm{Sh}}^{\mathrm{ad}}=\left(\Gamma_{\mathrm{Sh}}^{\mathrm{ad}}\right)^{2} \backslash U_{\mathrm{Sh}}^{\mathrm{ad}}$.

6.3. The map $\Theta: Z_{K} \rightarrow Z_{\mathbf{S h}}^{\text {ad }}$. The aim in this section is to construct a continuous map $\Theta: Z_{K} \rightarrow Z_{\mathrm{Sh}}^{\mathrm{ad}}$.

6.3.1. Relating $\boldsymbol{Z}_{\boldsymbol{K}}$ and $\boldsymbol{Z}_{\mathrm{Sh}}$. Recall that the morphism of Shimura data $\varphi: \delta_{K} \rightarrow$ $\delta_{\mathrm{Sh}}$ constructed in Section 3.3 is a morphism of algebraic groups $\varphi: T^{K} \rightarrow \mathrm{GSp}$, inducing a morphism $\operatorname{Sh}(\varphi): \operatorname{Sh}\left(\delta_{K}\right) \rightarrow \operatorname{Sh}\left(\delta_{\mathrm{Sh}}\right)$ of Shimura varieties. Moreover there is a natural continuation of $\varphi$ to a morphism of algebraic semigroups $M(\varphi): M^{K} \rightarrow$ MSp due to the following. We know that $\varphi: T^{K} \rightarrow$ GSp can be expressed in terms of reflex norms (see Lemmas 3.3 and B.3) that are given by determinants, and this definition makes still sense if we replace $T^{K}$ and GSp by their enveloping semigroups $M^{K}$ and MSp, respectively. Now we can define an equivariant morphism of topological groupoids

$$
\Omega: U_{K} \rightarrow U_{\mathrm{Sh}}
$$

by

$$
(g, m, z) \in U_{K} \mapsto\left(\varphi\left(\mathbb{A}_{f}\right)(g), M(\varphi)\left(\mathbb{A}_{f}\right)(z), \operatorname{Sh}(\varphi)(z)\right) \in U_{\mathrm{Sh}} .
$$


To show the equivariance of $\Omega$ use $\varphi\left(\mathbb{A}_{f}\right)\left(\Gamma_{K}\right) \subset \Gamma_{\mathrm{Sh}}$ and the equivariance of $\operatorname{Sh}(\varphi)$ (cf. D.2). We obtain a continuous map

$$
\bar{\Omega}: Z_{K} \rightarrow Z_{\mathrm{Sh}} .
$$

6.3.2. Relating $Z_{\mathrm{Sh}}$ and $Z_{\mathrm{Sh}}^{\text {ad }}$. In order to relate $Z_{\mathrm{Sh}}$ and $Z_{\mathrm{Sh}}^{\text {ad }}$ we will show that we are allowed to apply Criterion 5.1 by proving the following two lemmas.

Lemma 6.3. We have $\operatorname{GSp}\left(V_{E}, \psi_{E}\right)\left(\mathbb{A}_{f}\right)=\operatorname{GSp}\left(V_{E}, \psi_{E}\right)(\mathbb{Q}) \cdot \Gamma_{\mathrm{Sh}}$.

Proof. Let $\left\{e_{j}\right\}$ be an integral symplectic basis of $V_{E}$ with respect to $L_{E}$ (cf. Lemma 6.2). Each $f \in \operatorname{GSp}\left(\mathbb{A}_{f}\right)$ is $\mathbb{A}_{f}$-linear and therefore determined by the values on $e_{j} \otimes 1 \otimes 1 \in L_{E} \otimes_{\mathbb{Z}} \mathbb{Q} \otimes_{\mathbb{Z}} \hat{\mathbb{Z}}$ given by

$$
f\left(e_{j} \otimes 1 \otimes 1\right)=\sum_{k} a_{k, j} \otimes b_{k, j} \otimes c_{k, j} \in L_{E} \otimes_{\mathbb{Z}} \mathbb{Q} \otimes_{\mathbb{Z}} \hat{\mathbb{Z}} .
$$

Let $d_{k, j} \in \mathbb{N}$ be the denominator of $b_{k, j}$ and define $c(f)=\prod_{k, j} d_{k, j} \in \mathbb{N}$.

Now observe that the map

$$
M_{f}: e_{i} \mapsto c(f) e_{i}
$$

is a map in $\operatorname{GSp}(\mathbb{Q}) \subset \mathrm{GSp}\left(\mathbb{A}_{f}\right)$, i.e., $M_{f}$ is compatible with the symplectic structure $\psi_{E}$. Obviously

$$
M_{f} \circ f \in \Gamma_{\mathrm{Sh}},
$$

and thus we obtain the desired decomposition

$$
f=M_{f}^{-1} \circ\left(M_{f} \circ f\right) \in \mathrm{GSp}(\mathbb{Q}) \cdot \Gamma_{\mathrm{Sh}} .
$$

Lemma 6.4. The map $\mathrm{GSp}(\mathbb{Q}) \cap \Gamma_{\mathrm{Sh}} \rightarrow \mathrm{GSp}(\mathbb{Q}) / \mathrm{GSp}(\mathbb{Q})^{+}$is surjective.

Proof. We know that $\mathrm{GSp}(\mathbb{R})^{+}=\{f \in \mathrm{GSp}(\mathbb{R}) \mid v(f)>0\}$ (see A.2). From this we get $\mathrm{GSp}(\mathbb{Q})^{+}=\mathrm{GSp}(\mathbb{Q}) \cap \mathrm{GSp}(\mathbb{R})^{+}=\{f \in \mathrm{GSp}(\mathbb{Q}) \mid v(f)>0\}$.

Let $f$ be an element in $\mathrm{GSp}(\mathbb{Q})$. If we define $M_{f}$ exactly as in the proof above, we see that $M_{f} \in \mathrm{GSp}(\mathbb{Q})^{+}$and conclude that $M_{f} \circ f \in \mathrm{GSp}(\mathbb{Q}) \cap \Gamma_{\mathrm{Sh}}$.

Thus we see that the natural morphism $Z_{\mathrm{Sh}}^{+} \rightarrow Z_{\mathrm{Sh}}$ (see (27)) is a homeomorphism so that we can invert this map and compose it with the natural map $Z_{\mathrm{Sh}}^{+} \rightarrow Z_{\mathrm{Sh}}^{\mathrm{ad}}$ (cf. (29)) to obtain a continuous map

$$
Z_{\mathrm{Sh}} \rightarrow Z_{\mathrm{Sh}}^{\mathrm{ad}}
$$

Finally, if we compose the last map with $\bar{\Omega}$ from above we obtain a continuous denoted by

$$
\Theta: Z_{K} \rightarrow Z_{\mathrm{Sh}}^{\mathrm{ad}}
$$


One crucial property of $\Theta$ is that every element $z \in Z_{K}$ will be sent to an element of the form

$$
\Theta(z)=\left[g \beta^{-1}, \beta \rho, \alpha^{-1} x_{\mathrm{cm}}\right] \in Z_{\mathrm{Sh}}^{\mathrm{ad}},
$$

where $g \in G^{\mathrm{ad}}(\mathbb{Q})^{+}, \rho \in \Gamma_{\mathrm{Sh}, M}^{\mathrm{ad}}, \alpha \in G^{\mathrm{ad}}(\mathbb{Q})$ and $\beta \in \Gamma_{\mathrm{Sh}}^{\mathrm{ad}}$ such that $\alpha \beta \in$ $\pi^{\mathrm{ad}}\left(\varphi\left(T^{K}\left(\mathbb{A}_{f}\right)\right)\right) \subset G^{\mathrm{ad}}\left(\mathbb{A}_{f}\right)$.

\section{Construction of our arithmetic subalgebra}

The idea of the construction to follow goes back to [CMR05] and [CMR06].

We constructed the ring $\mathcal{M}^{\mathrm{cm}}$ of arithmetic modular functions on $\mathbb{H}_{g}$ that are defined in $x_{\mathrm{cm}}$ (see Section 4.3.1). Further the group $\overline{\mathcal{E}}$ acts by automorphisms on $\mathcal{M}^{\mathrm{cm}}$ according to Section 4.3.2. (Recall that we use the notation ${ }^{\alpha} f$ to denote the action of an automorphism $\alpha$ on a function $f \in \mathcal{M}^{\mathrm{cm}}$.) Thanks to the embeddings (cf. Section 4.3)

$$
\overline{\mathcal{E}} \rightarrow \frac{\mathrm{GSp}\left(\mathbb{A}_{f}\right)}{\mathbb{Q}^{\times}} \rightarrow \frac{\operatorname{MSp}\left(\mathbb{A}_{f}\right)}{\mathbb{Q}^{\times}} \leftarrow \Gamma_{\mathrm{Sh}, M}^{\mathrm{ad}}
$$

the intersection $\overline{\mathcal{E}} \cap \Gamma_{\mathrm{Sh}, M}^{\mathrm{ad}}$ is meaningful and thus we can define, for each $f \in \mathcal{M}^{\mathrm{cm}}$, a function $\tilde{f}$ on $U_{\mathrm{Sh}}^{\mathrm{ad}}$ by

$$
\tilde{f}(g, \rho, z)= \begin{cases}\rho f(z) & \text { if } \rho \in \overline{\mathcal{E}} \cap \Gamma_{\mathrm{Sh}, M}^{\mathrm{ad}}, \\ 0 & \text { else. }\end{cases}
$$

By construction $\tilde{f}$ is invariant under the action of $\left(\gamma_{1}, \gamma_{2}\right) \in \Gamma_{\mathrm{Sh}}^{\mathrm{ad}} \times \Gamma_{\mathrm{Sh}}^{\mathrm{ad}}$ because

$$
\tilde{f}\left(\gamma_{1} g \gamma_{2}^{-1}, \gamma_{2} m, \gamma_{2} z\right) \stackrel{\text { def }}{=} \gamma_{2} m f\left(\gamma_{2} z\right) \stackrel{(21)}{=} \gamma_{2}^{-1} \gamma_{2} m f(z)=\tilde{f}(g, m, z) .
$$

Therefore we can regard $\tilde{f}$ as function on the quotient $Z_{\mathrm{Sh}}^{\mathrm{ad}}=\left(\Gamma_{\mathrm{Sh}}^{\mathrm{ad}}\right)^{2} \backslash U_{\mathrm{Sh}}^{\mathrm{ad}}$.

We set $W_{K}=\hat{\mathcal{O}}_{K}^{\times} \times \widehat{\mathcal{O}}_{K} \times \operatorname{Sh}\left(T^{K}, X_{K}\right)$, which is a compact and clopen subset of $U_{K}$ and invariant under the action of $\Gamma_{K}^{2}$, i.e., $\Gamma_{K}^{2} \cdot W_{K} \subset W_{K}$. With these preliminaries we have the following result.

Proposition 7.1. Let $f$ be a function in $\mathcal{M}^{\mathrm{cm}}$. Then $\tilde{f} \circ \Theta$ is contained in $C_{c}\left(Z_{K}\right)$, i.e.,

$$
\tilde{f} \circ \Theta \in H_{K} \subset A_{K} .
$$

Proof. As we have already observed in (31), the image of an element $z \in Z_{K}$ under $\Theta$ is of the form $\left[g \beta^{-1}, \beta \rho, \alpha^{-1} x_{\mathrm{cm}}\right] \in Z_{\mathrm{Sh}}^{\text {ad }}$. Therefore $f_{K}=\tilde{f} \circ \Theta$ is continuous. Since $\Theta$ is continuous, the action of $\overline{\mathcal{E}}$ is continuous and does not produce singularities at special points (see Theorem 4.3).

Let us now regard $f_{K}$ as $\Gamma_{K}^{2}$-invariant function on $U_{K}$. Due to $\bar{\varepsilon} \subset \frac{\operatorname{GSp}\left(\mathbb{A}_{f}\right)}{\mathbb{Q}^{x}}$ and (32) we see that the support of our function $f_{K}$ is contained in the clopen subset 
$\hat{\mathcal{O}}_{K}^{\times} \times \hat{\mathcal{O}}_{K}^{\times} \times \operatorname{Sh}\left(T^{K}, X_{K}\right) \subset U_{K}$. Using the compact subset $W_{K} \subset U_{K}$, the next easy lemma finishes the proof.

Lemma 7.2. Let $G$ be a topological group, $X$ be a topological $G$-space and $Y \subset X$ a compact, clopen subset such that $G Y \subset Y$. If we have a continuous, $G$-invariant function $f \in C^{G}(X)$ then $\left.f\right|_{Y} \in C_{c}(G \backslash X)$.

Now we can define our arithmetic subalgebra of $\mathcal{A}_{K}=\left(A_{K}, \sigma_{t}\right)$ (cf. Section 6.1).

Definition 7.1. Denote by $A_{K}^{\text {arith }}$ the $K$-rational subalgebra of $A_{K}$ generated by the set of functions $\left\{\tilde{f} \circ \Theta \mid f \in \mathcal{M}^{\mathrm{cm}}\right\}$.

7.1. Proof of Theorem 1.1. Let $f \in \mathcal{M}^{\mathrm{cm}}$ and write $f_{K}=\tilde{f} \circ \Theta \in A_{K}^{\text {arith }}$. Further let us denote by $\varrho_{\omega}$ the extremal $\mathrm{KMS}_{\infty}$-state of $\mathcal{A}_{K}$ corresponding to $\omega \in$ $\mathrm{Sh}=\operatorname{Sh}\left(T^{K}, X_{K}, h_{K}\right)$, see Section 6.1.5. Recall the isomorphism $\operatorname{Gal}\left(K^{\mathrm{ab}} / K\right) \cong$ $\pi_{0}\left(C_{K}\right)=$ Sh given by Artin reciprocity. Considered as element in $\operatorname{Gal}\left(K^{\mathrm{ab}} / K\right)$ we write $[\omega]$ for $\omega$.

Property vi). Let $v \in \mathbb{A}_{K}^{\times}$be a symmetry of $\mathcal{A}_{K}$ (see Section 6.1.4). Due to Lemmas 6.2 and 6.3, we can write $\varphi\left(\mathbb{A}_{f}\right)(v)=\alpha \beta \in \mathrm{GSp}\left(\mathbb{A}_{f}\right)$ with $\alpha \in \mathrm{GSp}(\mathbb{Q})^{+}$ and $\beta \in \Gamma_{\mathrm{Sh}}$. By $\bar{\alpha}$ resp. $\bar{\beta}$ we will denote their images in $\mathrm{GSp}^{\text {ad }}(\mathbb{Q})^{+}$resp. $\Gamma_{\mathrm{Sh}}^{\text {ad }}$ under the map $\pi^{\text {ad }} \circ \varphi\left(\mathbb{A}_{f}\right)$. Moreover we denote the image of $v$ under Artin reciprocity by $[v] \in \operatorname{Gal}\left(K^{\mathrm{ab}} / K\right)$.

The action of the symmetries on the extremal $\mathrm{KMS}_{\infty}$-states is given by pull back and because this action is free and transitive it is enough to restrict to the case of the extremal $\mathrm{KMS}_{\infty}$-state $\varrho_{1}$ corresponding to the identity in $\mathrm{Gal}\left(K^{\mathrm{ab}} / K\right)$.

Using Proposition 4.4 and the reciprocity law (24) we can calculate the action of $v$ on $\varrho_{\omega}\left(f_{K}\right)$ as follows:

$$
\begin{aligned}
& { }^{v} \varrho_{1}\left(f_{K}\right) \stackrel{\text { def }}{=} \varrho_{1}\left({ }^{v} f_{K}\right) \\
& \stackrel{(31)}{=} \tilde{f}\left(\bar{\beta}^{-1}, \bar{\beta}, \bar{\alpha}^{-1} x_{\mathrm{cm}}\right) \\
& \text { Prop. } \\
& \stackrel{4.4}{=} \bar{\eta}(\beta) f\left(\bar{\alpha}^{-1} x_{\mathrm{cm}}\right) \\
& \stackrel{(21)}{=} \bar{\eta}(\alpha) \bar{\eta}(\beta) f\left(x_{\mathrm{cm}}\right) \\
& ={ }^{\bar{\eta}(v)} f\left(x_{\mathrm{cm}}\right) \\
& \stackrel{(24)}{=}[v]^{-1}\left(f\left(x_{\mathrm{cm}}\right)\right) \\
& =[v]^{-1}\left(\varrho_{1}\left(f_{K}\right)\right) \text {. }
\end{aligned}
$$

This is precisely the intertwining property we wanted to prove. 
Property v). Using the notation from above, we conclude immediately that by construction and Theorem 4.2 we have

$$
\varrho_{1}\left(f_{K}\right)=\tilde{f}\left(1,1, x_{x m}\right)=f\left(x_{\mathrm{cm}}\right) \in K^{\mathrm{ab}},
$$

and the above calculation shows further that

$$
\varrho_{\omega}\left(f_{K}\right)={ }^{[\omega]} \varrho_{1}\left(f_{K}\right)=[\omega]^{-1}\left(f\left(x_{\mathrm{cm}}\right)\right) \in K^{\mathrm{ab}},
$$

finishing our proof.

Remark. We want to conclude our paper with a short discussion comparing our construction with the original construction of Connes, Marcolli and Ramachdran (see [CMR05]) in the case of an imaginary quadratic field $K$. Apart from the fact that we are not dealing with the " $K$-lattice" picture as done in [CMR05] the main difference lies in the different definitions of symmetries. If the class number $h_{K}$ of $K$ is equal to one, it is immediate that the two definitions agree, however for $h_{K}>1$ their symmetries contain endomorphisms (see [CMR05], Proposition 2.17), whereas our symmetries are always given by automorphisms. We want to mention that it is no problem to generalize their definition to the context of a BC-type system for an arbitrary number field (this is already contained in [CM08]), and, without changing the definition of our arithmetic subalgebra, we could have proved Theorem 1.1 by using the new definition of symmetries (now containing endomorphisms).

This might look odd at first sight, but in the context of endomotives (see [CM08] for a reference) the relationship between the two different definitions will become transparent. More precisely, in a forthcoming article (cf. [Ya]) we will show that every BC-type system (for an arbitrary number field) is an endomotive, and the precise relationship between the two definitions of symmetries (and their actions on (extremal) $\mathrm{KMS}_{\beta}$-states) will become clear.

\section{Appendices}

\section{A. Algebraic groups}

Our references are [Wat79] and [Mil06]. Let $k$ denote a field of characteristic zero, $K$ a finite field extension of $k$ and $\bar{k}$ an algebraic closure of $k$. Further we denote by $R$ a $k$-algebra.

A.1. Functorial definition and basic constructions. An (affine) algebraic group $G$ (over $k$ ) is a representable functor from (commutative, unital) $k$-algebras to groups. We denote by $k[G]$ its representing algebra, i.e., for any $R$ we have $G(R)=$ $\operatorname{Hom}_{\mathrm{k}-\text { alg }}(k[G], R)$. 
A homomorphism $F: G \rightarrow H$ between two algebraic groups $G$ and $H$ (over $k$ ) is given by a natural transformation of functors.

Let $G$ and $H$ be two algebraic groups over $k$, then their direct product $G \times H$ is the algebraic group (over $k$ ) given by $R \mapsto G(R) \times H(R)$.

Let $G$ be an algebraic group over $k$ and $K$ and extension of $k$. Then by extension of scalars we obtain an algebraic group $G_{K}$ over $K$ represented by $K \otimes_{k} k[G]$.

Now let $G$ be an algebraic group over $K$. The Weil restriction $\operatorname{Res}_{K / k}(G)$ is an algebraic group over $k$ defined by $\operatorname{Res}_{K / k}(G)(R)=G\left(K \otimes_{k} R\right)$.

Remark. All three constructions are functorial.

A.2. Examples. 1) The multiplicative group $\mathbb{G}_{m, k}$ (over $k$ ) is represented by $k\left[x, x^{-1}\right]=k[x, y] /(x y-1)$, i.e., $\mathbb{G}_{m, k}(R)=R^{\times}$.

2) Define $\mathbb{S}=\operatorname{Res}_{\mathbb{C} / \mathbb{R}}\left(\mathbb{G}_{m}, \mathbb{C}\right)$. We have $\mathbb{S}(\mathbb{R})=\mathbb{C}^{\times}$and $\mathbb{\$}(\mathbb{C}) \cong \mathbb{C}^{\times} \times \mathbb{C}^{\times}$. In particular we have $\mathbb{S}_{\mathbb{C}} \cong \mathbb{G}_{m, \mathbb{C}} \times \mathbb{G}_{m, \mathbb{C}}$.

3) More general an algebraic group $T$ over $k$ is called a torus if $T_{\vec{k}}$ is isomorphic to a product of copies of $\mathbb{G}_{m, \bar{k}}$.

4) The general symplectic group $\operatorname{GSp}(V, \psi)$ attached to a symplectic $\mathbb{Q}$-vector space $(V, \psi)$ is an algebraic group over $\mathbb{Q}$ defined on a $\mathbb{Q}$-algebra $R$ by

$$
\begin{aligned}
& \operatorname{GSp}(R)=\left\{f \in \operatorname{End}_{R}\left(V \otimes_{\mathbb{Q}} R\right) \mid \text { there exists } v(f) \in R^{\times}\right. \text {such that } \\
& \left.\qquad \psi_{R}(f(x), f(y))=v(f) \psi_{R}(x, y) \text { for all } x, y \in V \otimes_{\mathbb{Q}} R\right\} .
\end{aligned}
$$

A.3. Characters. Let $G$ be an algebraic group over $k$ and set $\Lambda=\mathbb{Z}[\mathrm{Gal}(\bar{k} / k)]$. The character group $X^{*}(G)$ of $G$ is defined by $\operatorname{Hom}\left(G_{\bar{k}}, \mathbb{G}_{m, \bar{k}}\right)$. There is a natural action of $\operatorname{Gal}(\bar{k} / k)$ on $X^{*}(G)$, i.e., $X^{*}(G)$ is a $\Lambda$-module. Analogously the cocharacter group $X_{*}(G)$ of $G$ is the $\Lambda$-module $\operatorname{Hom}\left(\mathbb{G}_{m, \bar{k}}, G_{\bar{k}}\right)$. We denote the action of $\sigma \in \Lambda$ on a (co)character $f$ by $\sigma f$ or $f^{\sigma}$. The following is important.

Theorem A.1 ([Wat79], Chap. 7.3). The functor $G \mapsto X^{*}(G)$ is a contravariant equivalence from the category of algebraic groups of multiplicative type over $k$ and the category of finitely generated abelian groups with a continuous action of $\mathrm{Gal}(\bar{k} / k)$.

Remark. See [Wat79], Chap. 7.2, for the definition of groups of multiplicative type. We only have to know that algebraic tori are of multiplicative type.

There is a natural bi-additive and $\operatorname{Gal}(\bar{k} / k)$-invariant pairing $\langle\cdot, \cdot\rangle: X_{*}(G) \times$ $X^{*}(G) \rightarrow \mathbb{Z}$ given by $\langle\chi, \mu\rangle=\mu \circ \chi \in \operatorname{Hom}\left(\mathbb{G}_{m, \bar{k}}, \mathbb{G}_{m, \bar{k}}\right) \cong \mathbb{Z}$. If $G$ is of multiplicative type the pairing is perfect, i.e., there is an isomorphism of $\Lambda$-modules $X_{*}(G) \cong \operatorname{Hom}\left(X^{*}(G), \mathbb{Z}\right)$. 
A.4. Norm maps. Let $L$ be a finite field extension of $K$, i.e., we have a tower $k \subset K \subset L$, and let $T$ be a torus over $k$. Then there are two types of morphisms of algebraic groups which we call norm maps. The first one

$$
\mathrm{Nm}_{L / K}: \operatorname{Res}_{L / K}\left(T_{L}\right) \rightarrow T_{K}
$$

is induced by the usual norm map of algebras $R \otimes_{K} L \rightarrow R$ for $R$ a $K$-algebra. In applying the Weil restriction functor $\operatorname{Res}_{K / k}$ we obtain the second one, namely

$$
\mathrm{N}_{L / K}: \operatorname{Res}_{L / k}\left(T_{L}\right) \rightarrow \operatorname{Res}_{K / k}\left(T_{K}\right) .
$$

A.5. The case of number fields. Let $K$ be a number field. We are interested in the algebraic group $T^{K}=\operatorname{Res}_{K / \mathbb{Q}}\left(\mathbb{G}_{m, K}\right)($ over $\mathbb{Q})$. We have $T^{K}(R)=\left(K \otimes_{\mathbb{Q}} R\right)^{\times}$.

It is easy to see that the isomorphism $K \otimes \mathbb{Q} \overline{\mathbb{Q}} \cong \prod_{\rho \in \operatorname{Hom}(K, \overline{\mathbb{Q}})} \overline{\mathbb{Q}}$ induces an isomorphism of algebraic groups $T_{\overline{\mathbb{Q}}}^{K} \cong \prod_{\rho \in \operatorname{Hom}(K, \overline{\mathbb{Q}})} \mathbb{G}_{m, \overline{\mathbb{Q}}}$. It follows that $X^{*}\left(T^{K}\right) \cong$ $\mathbb{Z}^{\operatorname{Hom}(K, \overline{\mathbb{Q}})}$ with $\operatorname{Gal}(\overline{\mathbb{Q}} / \mathbb{Q})$ acting as follows. For $f=\sum_{\rho \in \operatorname{Hom}(K, \overline{\mathbb{Q}})} a_{\rho}[\rho] \in$ $\mathbb{Z}^{\mathrm{Hom}(K, \overline{\mathbb{Q}})}$ and $\sigma \in \operatorname{Gal}(\overline{\mathbb{Q}} / \mathbb{Q})$ we have

$$
\sigma f=\sum_{\rho \in \operatorname{Hom}(K, \overline{\mathbb{Q}})} a_{\rho}[\sigma \circ \rho]=\sum_{\rho \in \operatorname{Hom}(K, \overline{\mathbb{Q}})} a_{\sigma^{-1} \circ \rho}[\rho] .
$$

For any inclusion $K \subset L$ of number fields the norm map $\mathrm{N}_{L / K}: T^{L} \rightarrow T^{K}$ is defined by saying that a character $f=\sum_{\rho \in \operatorname{Hom}(K, \overline{\mathbb{Q}})} a_{\rho}[\rho] \in X^{*}\left(T^{K}\right)$ is mapped to the character $f_{L}=\sum_{\rho^{\prime} \in \operatorname{Hom}(L, \overline{\mathbb{Q}})}\left(a_{\rho^{\prime} \mid K}\right)\left[\rho^{\prime}\right] \in X^{*}\left(T^{L}\right)$.

\section{B. CM fields}

We follow [Mil06] and [Mil04]. By $\iota$ we denote the complex conjugation of $\mathbb{C}$.

B.1. CM fields and CM types. Let $E$ denote a number field. If $\mathrm{E}$ is a totally imaginary quadratic extension of a totally real field, we call $E$ a $C M$ field. In particular the degree of a CM field is always even. A $C M$ type $(E, \Phi)$ is a CM field $E$ together with a subset $\Phi \subset \operatorname{Hom}(E, \mathbb{C})$ such that $\Phi \cup \iota \Phi=\operatorname{Hom}(E, \mathbb{C})$ and $\Phi \cap \iota \Phi=\emptyset$.

B.2. About $\boldsymbol{h}_{\boldsymbol{\phi}}$ and $\boldsymbol{\mu}_{\boldsymbol{\phi}}$. Let $(E, \Phi)$ be a CM type. Then there are natural isomorphisms $T_{\mathbb{R}}^{E} \cong \prod_{\phi \in \Phi} \Phi$ resp. $T_{\mathbb{C}}^{E} \cong \prod_{\phi \in \Phi} \mathbb{G}_{m, \mathbb{C}} \times \prod_{\bar{\phi} \in \iota \Phi} \mathbb{G}_{m, \mathbb{C}}$, where the first one is induced by $E \otimes \mathbb{Q} \mathbb{R} \cong \prod_{\phi \in \Phi} \mathbb{C}$ and the second one by $E \otimes \mathbb{C} \cong$ $\prod_{\phi \in \Phi} \mathbb{C} \times \prod_{\bar{\phi} \in \iota \Phi} \mathbb{C}$.

Thus we obtain natural morphisms

$$
h_{\Phi}: \$ \rightarrow T_{\mathbb{R}}^{E}, \quad z \mapsto(z)_{\phi \in \Phi},
$$


and

$$
\mu_{\Phi}: \mathbb{G}_{m, \mathbb{C}} \rightarrow T_{\mathbb{C}}^{E}, \quad z \mapsto(z)_{\phi \in \Phi} \times(1)_{\bar{\phi} \in \iota \Phi}
$$

If we take $\mu_{\Phi}$ for granted we could have defined $h_{\Phi}$ by the composition

$$
\operatorname{Res}_{\mathbb{C} / \mathbb{R}}\left(\mathbb{G}_{m, \mathbb{C}}\right) \stackrel{\operatorname{Res}_{\mathbb{C} / \mathbb{R}}\left(\mu_{\Phi}\right)}{\longrightarrow} \operatorname{Res}_{\mathbb{C} / \mathbb{R}}\left(T_{\mathbb{C}}^{E}\right) \stackrel{\mathrm{Nm}_{\mathbb{C} / \mathbb{R}}}{\longrightarrow} T_{\mathbb{R}}^{E}
$$

In particular we see that $h_{\Phi}$ and $\mu_{\Phi}$ are related by

$$
h_{\Phi, \mathbb{C}}(z, 1)=\mu_{\Phi}(z) .
$$

Remark. In the last two sections one might have replaced $\mathbb{C}$ by $\overline{\mathbb{Q}}$.

B.3. The reflex field and reflex norm. Let $(E, \Phi)$ be a $C M$ type. The reflex field $E^{*}$ of $(E, \Phi)$ is the subfield of $\overline{\mathbb{Q}}$ defined by any one of the following conditions:

(a) $\sigma \in \operatorname{Gal}(\overline{\mathbb{Q}} / \mathbb{Q})$ fixes $E^{*}$ if and only if $\sigma \Phi=\Phi$;

(b) $E^{*}$ is the field generated over $\mathbb{Q}$ by the elements $\sum_{\phi \in \Phi} \phi(e), e \in E$;

(c) $E^{*}$ is the smallest subfield of $\overline{\mathbb{Q}}$ such that there exists a $E \otimes_{\mathbb{Q}} E^{*}$-module $V$ such that

$$
\operatorname{Tr}_{E^{*}}(e \mid V)=\sum_{\phi \in \Phi} \phi(e) \quad \text { for all } e \in E
$$

The reflex norm of $(E, \Phi)$ is the morphism of algebraic groups $\mathrm{N}_{\Phi}: T^{E^{*}} \rightarrow T^{E}$ given, for $R$ a $\mathbb{Q}$-algebra, by

$$
a \in T^{E^{*}}(R) \mapsto \operatorname{det}_{E \otimes_{\mathbb{Q}} R}\left(a \mid V \otimes_{\mathbb{Q}} R\right) \in T^{E}(R) .
$$

\section{The Serre group}

Our references are [Mil98], [Mil06] and [Wei]. Let $K$ be a number field. We fix an embedding $\tau: K \rightarrow \overline{\mathbb{Q}} \rightarrow \mathbb{C}$ and denote by $\iota$ complex conjugation on $\mathbb{C}$.

C.1. Definition of the Serre group. The following are equivalent:

(1) The Serre group attached to $K$ is a pair $\left(S^{K}, \mu^{K}\right)$ consisting of a $\mathbb{Q}$-algebraic torus $S^{K}$ and a cocharacter $\mu^{K} \in X_{*}\left(S^{K}\right)$ defined by the following universal property. For every pair $(T, \mu)$ consisting of a $\mathbb{Q}$-algebraic torus $T$ and a cocharacter $\mu \in X_{*}(T)$ defined over $K$ satisfying the Serre condition,

$$
(\iota+1)(\sigma-1) \mu=0=(\sigma-1)(\iota+1) \mu \text { for all } \sigma \in \operatorname{Gal}(\overline{\mathbb{Q}} / \mathbb{Q}),
$$


there exists a unique morphism $\rho_{\mu}: S^{K} \rightarrow T$ such that the diagram

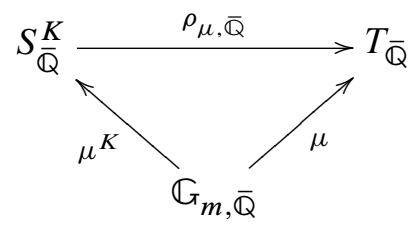

commutes.

(2) The Serre group $S^{K}$ is defined to be the quotient of $T^{K}$ such that $X^{*}\left(S^{K}\right)$ is the subgroup of $X^{*}\left(T^{K}\right)$ given by all elements $f \in X^{*}\left(T^{K}\right)$ which satisfy the Serre condition

$$
(\sigma-1)(\iota+1) f=0=(\iota+1)(\sigma-1) f \text { for all } \sigma \in \operatorname{Gal}(\overline{\mathbb{Q}} / \mathbb{Q}) .
$$

The cocharacter $\mu^{K}$ is induced by the cocharacter $\mu_{\tau} \in X_{*}\left(T^{K}\right)$ defined by

$$
\left\langle\mu_{\tau}, \Sigma n_{\sigma}[\sigma]\right\rangle=n_{\tau} \quad \text { for all } \Sigma n_{\sigma}[\sigma] \in \mathbb{Z}^{\operatorname{Hom}(K, \overline{\mathbb{Q}})} \cong X^{*}\left(T^{K}\right) .
$$

(3) If $K$ does not contain a CM subfield, we set $E=\mathbb{Q}$, otherwise $E$ denotes the maximal CM subfield of $K$ and $F$ the maximal totally real subfield of $E$. Then there is an exact sequence of $\mathbb{Q}$-algebraic groups

$$
1 \rightarrow \operatorname{ker}\left(\mathrm{N}_{F / \mathbb{Q}}: T^{F} \rightarrow T^{\mathbb{Q}}\right) \stackrel{i}{\rightarrow} T^{K} \stackrel{\pi^{K}}{\rightarrow} S^{K} \rightarrow 1,
$$

where $i$ is the obvious inclusion. The cocharacter $\mu_{\tau}$ of $T^{K}$, defined as in (2), induces $\mu^{K}$, i.e., $\mu^{K}=\pi^{K} \circ \mu_{\tau}$.

Remark. For $K=\mathbb{Q}$ or $K$ an imaginary quadratic field there is the obvious equality $S^{K}=T^{K}$.

C.2. About $\boldsymbol{\mu}^{K}$ and $\boldsymbol{h}^{K}$. The cocharacter $\mu^{K}=\pi^{K} \circ \mu_{\tau}: \mathbb{G}_{m, \mathbb{C}} \rightarrow S_{\mathbb{C}}^{K}$ from the last section induces a natural morphism

$$
h^{K}: \$ \rightarrow S_{\mathbb{R}}^{K}
$$

defined by

$$
\operatorname{Res}_{\mathbb{C} / \mathbb{R}}\left(\mathbb{G}_{m, \mathbb{C}}\right) \stackrel{\operatorname{Res}_{\mathbb{C} / \mathbb{R}}\left(\mu^{K}\right)}{\longrightarrow} \operatorname{Res} \mathbb{C}_{\mathbb{R}}\left(S_{\mathbb{C}}^{K}\right) \stackrel{\mathrm{Nm}_{\mathbb{C} / \mathbb{R}}}{\longrightarrow} S_{\mathbb{R}}^{K} .
$$

We see that $\mu^{K}$ and $h^{K}$ are related by

$$
h_{\mathbb{C}}^{K}(z, 1)=\mu^{K}(z)
$$

or in other words, for $z \in \mathbb{C}^{\times}$, we have $h^{K}(z)=\mu^{K}(z) \mu^{K}(z)^{\iota}$. 
C.3. About $\rho_{\boldsymbol{\Phi}}$ and the reflex norm $\mathbf{N}_{\boldsymbol{\Phi}}$. Let $(E, \Phi)$ be a CM type. The natural morphism $\mu_{\Phi} \in X_{*}\left(T^{E}\right)$ (cf. (34)) is defined over the reflex field $E^{*}$ and an easy calculation shows that it satisfies the Serre condition (35). By the universal property of the Serre group we obtain a $\mathbb{Q}$-rational morphism

$$
\rho_{\Phi}: S^{E^{*}} \rightarrow T^{E}
$$

such that

$$
\mu_{\Phi}=\rho_{\Phi, \mathbb{C}} \circ \mu^{E^{*}} .
$$

Also we see immediately that

$$
h_{\Phi}=\rho_{\Phi, \mathbb{R}} \circ h^{E^{*}} .
$$

Moreover we can relate $\rho_{\Phi}$ and $\mu_{\Phi}$ by the commutative diagram

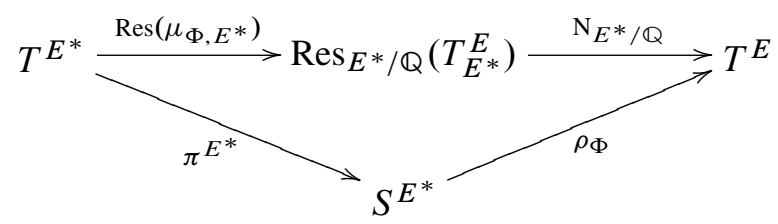

which can be seen on the level of characters. The relation with the reflex norm $\mathrm{N}_{\Phi}: T^{E^{*}} \rightarrow T^{E}$ (see B.3) is given by the following important result.

Proposition C.1 ([Mil06]). We have the equality

$$
\mathrm{N}_{\Phi}=\mathrm{N}_{E^{*} / \mathbb{Q}} \circ \operatorname{Res}\left(\mu_{\Phi, E^{*}}\right) .
$$

C.4. More properties of the Serre group. The following properties are all taken from [Mil06].

Proposition C.2. Let $E \subset K$ denote two number fields.

(1) The norm map $\mathrm{N}_{K / E}: T^{K} \rightarrow T^{E}$ induces a commutative diagram

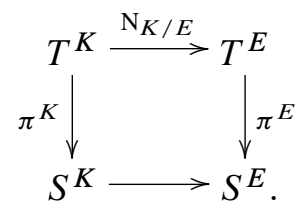

We call the induced morphism $\mathrm{N}_{K / E}: S^{K} \rightarrow S^{E}$.

(2) There is a commutative diagram

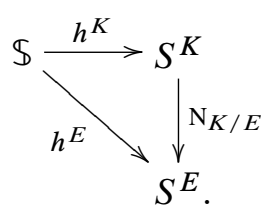


(3) Let E denote the maximal CM field contained in $K$, if there is no such subfield we set $E=\mathbb{Q}$. Then $\mathrm{N}_{K / E}: S^{K} \rightarrow S^{E}$ is an isomorphism.

(4) Let $(E, \Phi)$ be a CM type and $K_{1} \subset K_{2}$ two number fields, such that $E^{*} \subset K_{1}$, and let $\rho_{\Phi, i}: S^{K_{i}} \rightarrow T^{E}$ be the corresponding maps from the universal property of the Serre group. Then we have

$$
\rho_{\Phi, 1} \circ \mathrm{N}_{K_{2} / K_{1}}=\rho_{\Phi, 2}
$$

\section{Shimura varieties}

Our references are Deligne [Del79], Milne [Mil04], and Hida [Hid04].

Let $G$ be an algebraic group over $\mathbb{Q}$. Then the adjoint group $G^{\text {ad }}$ of $G$ is defined to be the quotient of $G$ by its center $C$. The derived group $G^{\text {der }}$ of $G$ is defined to be the intersection of the normal algebraic subgroups of $G$ such that $G / N$ is commutative. By $G(\mathbb{R})^{+}$we denote the identity component of $G(\mathbb{R})$ relative to its real topology and set $G(\mathbb{Q})^{+}=G(\mathbb{Q}) \cap G(\mathbb{R})^{+}$. If $G$ is reductive, we denote by $G(\mathbb{R})_{+}$the group of elements of $G(\mathbb{R})$ whose image in $G^{\text {ad }}(\mathbb{R})$ lies in its identity component and set $G(\mathbb{Q})_{+}=G(\mathbb{Q}) \cap G(\mathbb{R})_{+}$.

D.1. Shimura datum. A Shimura datum is a pair $(G, X)$ consisting of a reductive group $G\left(\right.$ over $\mathbb{Q}$ ) and a $G(\mathbb{R})$-conjugacy class $X$ of homomorphisms $h: \mathbb{S} \rightarrow G_{\mathbb{R}}$ such that the following (three) axioms are satisfied:

(SV1) For each $h \in X$, the representation $\operatorname{Lie}\left(G_{\mathbb{R}}\right)$ defined by $h$ is of type $\{(-1,1),(0,0),(1,-1)\}$.

(SV2) For each $h \in X, \operatorname{ad}(h(i))$ is a Cartan involution on $G_{\mathbb{R}}^{\text {ad }}$.

(SV3) $G^{\text {ad }}$ has no $\mathbb{Q}$-factors on which the projection of $h$ is trivial.

Since $G(\mathbb{R})$ acts transitively on $X$, it is enough to give a morphism $h_{0}: \mathbb{S} \rightarrow G_{\mathbb{R}}$ to specify a Shimura datum. Therefore a Shimura datum is sometimes written as triple $\left(G, X, h_{0}\right)$ or simply by $\left(G, h_{0}\right)$.

Further in our case of interest the following axioms are satisfied and (simplify the situation enormously).

(SV4) The weight homomorphism $\omega_{X}: \mathbb{G}_{m, \mathbb{R}} \rightarrow G_{\mathbb{R}}$ is defined over $\mathbb{Q}$.

(SV5) The group $C(\mathbb{Q})$ is discrete in $C\left(\mathbb{A}_{f}\right)$.

(SV6) The identity component of the center $C^{o}$ splits over a CM-field.

(SC) The derived group $G^{\text {der }}$ is simply connected.

(CT) The center $C$ is a cohomologically trivial torus.

Remark. (1) Axioms (SV1)-(SV6) are taken from [Mil04], the other two axioms are taken from [Hid04]. 
(2) The axioms of a Shimura variety (SV1)-(SV3) imply, for example, that $X$ is a finite union of hermitian symmetric domains. When viewed as an analytic space we sometimes write $x$ instead of $h$ for points in $X$ and $h_{x}$ for the associated morphism $h_{x}: \mathbb{S} \rightarrow G_{\mathbb{R}}$.

(3) In [HP05], Definition 3.1, a more general definition of a Shimura datum is given. For our purpose Deligne's original definition, as given above, and so-called 0 -dimensional Shimura varieties are sufficient.

A morphism of Shimura data $(G, X) \rightarrow\left(G^{\prime}, X^{\prime}\right)$ is a morphism $G \rightarrow G^{\prime}$ of algebraic groups which induces a map $X \rightarrow X^{\prime}$.

D.2. Shimura varieties. Let $(G, X)$ be a Shimura datum and let $K$ be a compact open subgroup of $G\left(\mathbb{A}_{f}\right)$. Set $\operatorname{Sh}_{K}=\operatorname{Sh}_{K}(G, X)=G(\mathbb{Q}) \backslash X \times G\left(\mathbb{A}_{f}\right) / K$, where $G(\mathbb{Q})$ is acting on $X$ and $G\left(\mathbb{A}_{f}\right)$ on the left, and $K$ is acting on $G\left(\mathbb{A}_{f}\right)$ on the right. On can show (see [Mil04], Lemma 5.13) that there is a homeomorphism $\mathrm{Sh}_{K} \cong \bigsqcup \Gamma_{g} \backslash X^{+}$. Here $X^{+}$is a connected component of $X$ and $\Gamma_{g}$ is the subgroup $g K g^{-1} \cap G(\mathbb{Q})_{+}$where $g$ runs over a set of representatives of $G(\mathbb{Q})_{+} \backslash G\left(\mathbb{A}_{f}\right) / K$. When $K$ is chosen sufficiently small, then $\Gamma_{g} \backslash X^{+}$is an arithmetic locally symmetric variety. For an inclusion $K^{\prime} \subset K$ we obtain a natural map $\mathrm{Sh}_{K^{\prime}} \rightarrow \mathrm{Sh}_{K}$ and in this way an inverse system $\left(\mathrm{Sh}_{K}\right)_{K}$. There is a natural right action of $G\left(\mathbb{A}_{f}\right)$ on this system (cf. [Mil04], p. 307).

The Shimura variety $\operatorname{Sh}(G, X)$ associated with the Shimura datum $(G, X)$ is defined to be the inverse limit of varieties $\lim _{K} \operatorname{Sh}_{K}(G, X)$ together with the natural action of $G\left(\mathbb{A}_{f}\right)$. Here $K$ runs through sufficiently small compact open subgroups of $G\left(\mathbb{A}_{f}\right) . \operatorname{Sh}(G, X)$ can be regarded as a scheme over $\mathbb{C}$.

Let $(G, X)$ be a Shimura datum such that (SV5) holds, then one has

$$
\operatorname{Sh}(G, X)=\lim _{K} \operatorname{Sh}_{K}(G, X)=G(\mathbb{Q}) \backslash X \times G\left(\mathbb{A}_{f}\right) .
$$

In this case we write $[x, l]$ for an element in $\operatorname{Sh}(G, X)$ and the (right) action of an element $g \in G\left(\mathbb{A}_{f}\right)$ is given by

$$
g[x, l]=[x, l g] .
$$

In the general case, when ( $S V 5)$ is not holding we use the same notation, understanding that $[x, l]$ stands for a family $\left(x_{K}, l_{K}\right)_{K}$ indexed by compact open subgroups $K$ of $G\left(\mathbb{A}_{f}\right)$.

A morphism of Shimura varieties $\operatorname{Sh}(G, X) \rightarrow \operatorname{Sh}\left(G^{\prime}, X^{\prime}\right)$ is an inverse system of regular maps of algebraic varieties compatible with the action of $G\left(\mathbb{A}_{f}\right)$. We have the following functorial property:

A morphism $\varphi:(G, X) \rightarrow\left(G^{\prime}, X^{\prime}\right)$ of Shimura data defines an equivariant morphism $\operatorname{Sh}(\varphi): \operatorname{Sh}(G, X) \rightarrow \operatorname{Sh}\left(G^{\prime}, X^{\prime}\right)$ of Shimura varieties, which is a closed immersion if $G \rightarrow G^{\prime}$ is injective ([Mil04], Theorem 5.16). 
D.3. Example. We want to give some details about the Shimura varieties attached to the data $S_{\mathrm{Sh}}$ constructed in Section 3.2. For the identification of the GSp(R)conjugacy class of $h_{\mathrm{cm}}$ with the higher Siegel upper lower half space

$$
\mathbb{Q}_{g}^{ \pm}=\left\{M=A+i B \in M_{g}(\mathbb{C}) \mid A=A^{t}, B \text { positive or negative definitive }\right\}
$$

we refer further to [Mil04], Exercise 6.2, p. 316.

In addition the data $\delta_{\mathrm{Sh}}$ fulfill all the axioms stated in D.1. The validity of (SV1)-(SV6) is shown in [Mil04], p. 316, and the validity of (SC) and (CT) in [Hid04]. The latter two axioms are important for making the arguments in [MS81] in this case. From (SV5) follows in particular that we do not have to bother about the limits in the definition of $\operatorname{Sh}\left(\mathrm{GSp}, \mathbb{U}_{g}^{ \pm}\right.$) because we have

$$
\operatorname{Sh}\left(\mathrm{GSp}, \mathbb{I}_{g}^{ \pm}\right)=\mathrm{GSp}(\mathbb{Q}) \backslash\left(\mathbb{I}_{g}^{ \pm} \times \mathrm{GSp}\left(\mathbb{A}_{f}\right)\right) .
$$

D.4. Connected Shimura varieties. A connected Shimura datum is a pair $\left(G, X^{+}\right)$ consisting of a semisimple algebraic group $G$ over $\mathbb{Q}$ and a $G^{\text {ad }}(\mathbb{R})^{+}$-conjugacy class of homomorphisms $h: \mathbb{\$} \rightarrow G_{\mathbb{R}}^{\text {ad }}$ satisfying axioms (SV1)-(SV3).

The connected Shimura variety $\operatorname{Sh}^{o}\left(G, X^{+}\right)$associated with a connected Shimura datum $\left(G, X^{+}\right)$is defined by the inverse limit

$$
\operatorname{Sh}^{o}\left(G, X^{+}\right)=\lim _{\Gamma} \Gamma \backslash X^{+}
$$

where $\Gamma$ runs over the torsion-free arithmetic subgroups of $G^{\text {ad }}(\mathbb{Q})^{+}$whose inverse image in $G(\mathbb{Q})^{+}$is a congruence subgroup. If we start with a Shimura datum $(G, X)$ and choose a connected component $X^{+}$of $X$, we can view $X^{+}$as a $G^{\text {ad }}(\mathbb{R})^{+}$conjugacy class of morphisms $h: \mathbb{S} \rightarrow G_{\mathbb{R}}^{\text {ad }}$ by projecting elements in $X^{+}$to $G_{\mathbb{R}}^{\text {ad }}$. One can show that $\left(G^{\mathrm{der}}, X^{+}\right)$is a connected Shimura datum. Further, if we choose the connected component $\operatorname{Sh}(G, X)^{o}$ of $\operatorname{Sh}(G, X)$ containing $X^{+} \times 1$, one has the following compatibility relation

$$
\operatorname{Sh}(G, X)^{o}=\operatorname{Sh}^{o}\left(G^{\mathrm{der}}, X^{+}\right) .
$$

D.5. 0-dimensional Shimura varieties. In Section 3.1 we defined a "Shimura datum" $S_{K}=\left(T^{K}, X_{K}\right)$ which is not a Shimura datum in the above sense because $X_{K}$ has more than one conjugacy class (recall that $T^{K}$ is commutative). Rather $S_{K}$ is a Shimura datum in the generalized sense of Pink [Pin90], which we do not want to recall here. Instead we define the notion of a 0 -dimensional Shimura variety following [Mil04], which covers all exceptional Shimura data we consider. We define a 0 -dimensional Shimura datum to be a triple $(T, Y, h)$, where $T$ is a torus over $\mathbb{Q}, Y$ is a finite set on which $T(\mathbb{R}) / T(\mathbb{R})^{+}$acts transitively, and $h: \mathbb{S} \rightarrow T_{\mathbb{R}}$ is a morphism of algebraic groups. We view $Y$ as a finite cover of $\{h\}$. We remark that the axioms (SV1)-(SV3) are automatically satisfied in this setup. 
The associated 0-dimensional Shimura variety $\operatorname{Sh}(T, Y, h)$ is defined to be the inverse system of finite sets $T(\mathbb{Q}) \backslash Y \times T\left(\mathbb{A}_{f}\right) / K$, with $K$ running over the compact open subgroups of $T\left(\mathbb{A}_{f}\right)$.

A morphism $(T, Y, h) \rightarrow\left(H, h_{0}\right)$ from a 0 -dimensional Shimura datum to a Shimura datum, with $H$ an algebraic torus, is given by a morphism of algebraic groups $\varphi: T \rightarrow H$ such that $h=\varphi_{\mathbb{R}} \circ h_{0}$.

If $\varphi$ is such a morphism it defines a morphism $\operatorname{Sh}(\varphi): \operatorname{Sh}(T, Y, h) \rightarrow \operatorname{Sh}\left(H, h_{0}\right)$ of Shimura varieties.

Remark. We have that $S_{K}$ fulfills axiom (SV5) if and only if $K=\mathbb{Q}$ or $K$ an imaginary quadratic field (see [HP05], Example 3.2).

D.6. Canonical model of Shimura varieties. Let $(G, X)$ be a Shimura datum. A point $x \in X$ is called a special point if there exists a torus $T \subset G$ such that $h_{x}$ factors through $T_{\mathbb{R}}$. The pair $(T, x)$ or $\left(T, h_{x}\right)$ is called special pair. If $(G, X)$ satisfies the axioms (SV4) and (SV6), then a special point is called CM point and a special pair is called CM pair.

Now given a special pair $(x, T)$ we can consider the cocharacter $\mu_{x}$ of $G_{\mathbb{C}}$ defined by $\mu_{x}(z)=h_{x, \mathbb{C}}(z, 1)$. Denote by $E(x)$ the field of definition of $\mu_{x}$, i.e., the smallest subfield $k$ of $\mathbb{C}$ such that $\mu_{x}: \mathbb{G}_{m, k} \rightarrow G_{k}$ is defined.

Let $R_{x}$ denote the composition

$$
T^{E(x)} \stackrel{\operatorname{Res}_{E}(x) / \mathbb{Q}\left(\mu_{x}\right)}{\longrightarrow} \operatorname{Res}_{E(x) / \mathbb{Q}}\left(T_{E}(x)\right) \stackrel{\mathrm{Nm}_{E(x) / \mathbb{Q}}}{\longrightarrow} T
$$

and define the reciprocity morphism

$$
r_{x}=R_{x}\left(\mathbb{A}_{f}\right): \mathbb{A}_{E(x), f}^{\times} \rightarrow T\left(\mathbb{A}_{f}\right) .
$$

Moreover, every datum $(G, X)$ defines an algebraic number field $E(G, X)$, the reflex field of $(G, X)$. For the definition we refer the reader to [Mil04], Section 12.2.

Remark. (1) For the Shimura datum $\delta_{\mathrm{Sh}}=\left(\mathrm{GSp}, \mathbb{Q}_{g}^{ \pm}\right.$) (see Section 3.2) we have $E\left(\delta_{K}\right)=\mathbb{Q}$ (cf. [Mil04], p. 352).

(2) For explanations to relations with the reflex field of a CM field (cf. B.3), see [Mil04], Example 12.4 b), p. $344 \mathrm{ff}$.

A model $M^{o}(G, X)$ of $\operatorname{Sh}(G, X)$ over the reflex field $E(G, X)$ is called canonical if

(1) $M^{o}(G, X)$ is equipped with a right action of $G\left(\mathbb{A}_{f}\right)$ that induces an equivariant isomorphism $M^{o}(G, X)_{\mathbb{C}} \cong \operatorname{Sh}(G, X)$, and

(2) for every special pair $(T, x) \subset(G, X)$ and $g \in G\left(\mathbb{A}_{f}\right)$ the point $[x, g] \in$ $M^{o}(G, X)$ is rational over $E(x)^{\mathrm{ab}}$ and the action of $\sigma \in \operatorname{Gal}\left(E(x)^{\mathrm{ab}} / E(x)\right)$ is given by

$$
\sigma[x, g]=\left[x, r_{x}(v) g\right],
$$

where $v \in \mathbb{A}_{E(x), f}^{\times}$is such that $[v]=\sigma^{-1}$ under Artin's reciprocity map. 
In particular, for every compact open subgroup $K \subset G\left(\mathbb{A}_{f}\right)$ it follows that $M_{K}^{o}(G, X)=M^{o}(G, X) / K$ is a model of $\operatorname{Sh}_{K}(G, X)$ over $E(G, X)$.

Remark. Canonical models are known to exist for all Shimura varieties (see [Mil04]).

D.7. Canonical model of connected Shimura varieties. We refer to [Del79], 2.7.10, for the precise definition of the canonical model $M^{o}\left(G, X^{+}\right)$of a connected Shimura variety $\operatorname{Sh}\left(G, X^{+}\right)$. Here we just want to mention the compatibility

$$
M^{o}\left(G^{\mathrm{der}}, X^{+}\right)=M^{o}(G, X)^{o},
$$

where the latter denotes a correctly chosen connected component of the canonical model $M^{o}(G, X)$.

\section{References}

[BC95] J.-B. Bost and A. Connes, Hecke algebras, type III factors and phase transitions with spontaneous symmetry breaking in number theory. Selecta Math. (N.S.) 1 (1995), 411-457. Zbl 0842.46040 MR 1366621

[BR79] O. Bratteli and D. W. Robinson, Operator algebras and quantum statistical mechanics. Vol. 1, Texts Monographs Phys., Springer-Verlag, New York 1979. Zbl 0421.46048 MR 545651

[BR81] O. Bratteli and D. W. Robinson, Operator algebras and quantum-statistical mechanics. Vol. 2, Texts Monographs Phys., Springer-Verlag, New York 1981. Zbl 0463.46052 MR 611508

[CM08] A. Connes and M. Marcolli, Noncommutative geometry, quantum fields and motives. Amer. Math. Soc. Colloq. Publ. 55, Amer. Math. Soc., Providence, RI, 2008. Zbl 1209.58007 MR 2371808

[CMR05] A. Connes, M. Marcolli, and N. Ramachandran, KMS states and complex multiplication. Selecta Math. (N.S.) 11 (2005), 325-347. Zbl 1106.58005 MR 2215258

[CMR06] A. Connes, M. Marcolli, and N. Ramachandran, KMS states and complex multiplication. II. In Operator Algebras: The Abel Symposium 2004, Abel Symp. 1, Springer, Berlin 2006, 15-59. Zbl 1123.58004 MR 2265042

[Del79] P. Deligne, Variétés de Shimura: interprétation modulaire, et techniques de construction de modèles canoniques. In Automorphic forms, representations and Lfunctions (Proc. Sympos. Pure Math., Oregon State Univ., Corvallis, Ore., 1977), Part 2, Proc. Sympos. Pure Math. 33, Amer. Math. Soc., Providence, R.I., 1979, 247-289. Zbl 0437.14012 MR 546620

[Hid04] H. Hida, p-adic automorphic forms on Shimura varieties. Springer Monogr. Math., Springer-Verlag, New York 2004. Zbl 1055.11032 MR 2055355

[HP05] E. Ha and F. Paugam, Bost-Connes-Marcolli systems for Shimura varieties. I. Definitions and formal analytic properties. Int. Math. Res. Pap. 2005 (2005), 237-286. Zbl 1173.82305 MR 2199962 
[LLN09] M. Laca, N. S. Larsen, and S. Neshveyev, On Bost-Connes types systems for number fields. J. Number Theory 129 (2009),325-338. Zbl 1175.46061 MR 2473881

[Mil98] J.S. Milne, Abelian varieties with complex multiplication (for pedestrians). Preprint 1998. arXiv:math/9806172

[Mil04] J. S. Milne, Introduction to Shimura varieties. In Harmonic analysis, the trace formula, and Shimura varieties. Clay Math. Proc. 4, Amer. Math. Soc., Providence, RI, 2005, 265-378. Zbl 1148.14011 MR 2192012

[Mil06] J. S. Milne, Complex multiplication. Course notes, 2006. http://www.jmilne.org/math/CourseNotes/cm.html

[MS81] J. S. Milne and K.-y. Shih, Automorphism groups of Shimura varieties and reciprocity laws. Amer. J. Math. 103 (1981), 911-935. Zbl 0475.14022 MR 630772

[Pin90] R. Pink, Arithmetical compactification of mixed Shimura varieties. Ph. D. thesis, Bonn, 1989; Bonner Math. Schriften 209, Universität Bonn Mathematisches Institut, Bonn 1990. Zbl 0748.14007 MR 1128753

[PR94] V. Platonov and A. Rapinchuk, Algebraic groups and number theory. Pure Appl. Math. 139, Academic Press, Boston 1994. Zbl 0841.20046 MR 1278263

[Shi00] G. Shimura, Arithmeticity in the theory of automorphic forms. Math. Surveys Monogr. 82, Amer. Math. Soc., Providence, RI, 2000. Zbl 0967.11001 MR 1780262

[Sil94] J. H. Silverman, Advanced topics in the arithmetic of elliptic curves. Graduate Texts in Math. 151, Springer-Verlag, New York 1994. Zbl 0911.14015 MR 1312368

[Wat79] W. C. Waterhouse, Introduction to affine group schemes. Graduate Texts in Math. 66, Springer-Verlag, New York 1979. Zbl 0442.14017 MR 547117

[Wei] W. Wei, Moduli fields of CM motives applied to Hilbert's 12-th problem. Preprint 1994. http://www.mathematik.uni-bielefeld.de/sfb343/preprints/pr94070.ps.gz

[Ya] B. Yalkinoglu, On arithmetic models and functoriality of Bost-Connes systems. With an appendix by Sergey Neshveyev. Invent. Math., to appear.

Doi 10.1007/s00222-012-0396-1

Received May 19, 2010

B. Yalkinoglu, Institut de Mathématiques, Université Pierre et Marie Curie - Paris 6, 175 Rue du Chevaleret, 75013 Paris, France

E-mail: boray@math.jussieu.fr 